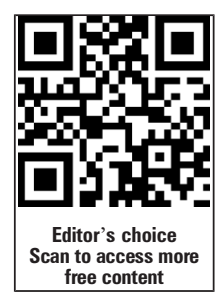

can to access m free content
- Additional material is published online only. To view please visit the journal online (http://dx.doi.org/10.1136/ gutjnl-2015-310318).

For numbered affiliations see end of article.

\section{Correspondence to}

Dr Hongyang Wang, International Co-operation Laboratory on Signal Transduction, Eastern Hepatobiliary Surgery Institute, Second Military Medical University, Shanghai 200438 ,

China; hywangk@vip.sina.com

Lei Chen, International Cooperation Laboratory on Signal Transduction, Eastern Hepatobiliary Surgery Institute, Second Military Medical University, 225 Changhai Road, Shanghai 200438, China; Chenlei@smmu.edu.cn

$Y C Y, X M L, X Y L$ and $G J L$ are co-first authors.

Received 8 July 2015 Revised 10 January 2016 Accepted 12 January 2016 Published Online First 9 February 2016

\section{SLinked}

- http://dx.doi.org/10.1136/ gutjnl-2016-311446

To cite: Yang $Y$, Lin $X$ Lu $X$, et al. Gut

2016;65:1186-1201.

\title{
Interferon-microRNA signalling drives liver precancerous lesion formation and hepatocarcinogenesis
}

\author{
Yingcheng Yang, ${ }^{1,2}$ Ximeng Lin, ${ }^{1,2}$ Xinyuan Lu, ${ }^{3}$ Guijuan Luo, ${ }^{1,4}$ Tao Zeng, $^{5}$ \\ Jing Tang, ${ }^{1,4}$ Feng Jiang, ${ }^{1}$ Liang Li, ${ }^{1,4}$ Xiuliang Cui, ${ }^{1,4}$ Wentao Huang, ${ }^{1}$ Guojun Hou, ${ }^{1}$ \\ Xin Chen, ${ }^{1}$ Qing Ouyang, ${ }^{1}$ Shanhua Tang, ${ }^{1}$ Huanlin Sun, ${ }^{1}$ Luonan Chen, ${ }^{5}$ \\ Frank J Gonzalez, ${ }^{6}$ Mengchao Wu, ${ }^{2}$ Wenming Cong, ${ }^{3}$ Lei Chen, ${ }^{1,4,6}$ Hongyang Wang ${ }^{1,4,7}$
}

\section{ABSTRACT}

Objective Precancerous lesion, a well-established histopathologically premalignant tissue with the highest risk for tumourigenesis, develops preferentially from activation of DNA damage checkpoint and persistent inflammation. However, little is known about the mechanisms by which precancerous lesions are initiated and their physiological significance.

Design Laser capture microdissection was used to acquire matched normal liver, precancerous lesion and tumour tissues. miR-484 $4^{-1-}$, Ifnar $1^{-1-}$ and Tgfbr2 $\triangle$ hep mice were employed to determine the critical role of the interferon (IFN)-microRNA pathway in precancerous lesion formation and tumourigenesis. RNA immunoprecipitation (RIP), pull-down and chromatin immunoprecipitation (ChIP) assays were applied to explore the underlying mechanisms.

Results miR-484 is highly expressed in over $88 \%$ liver samples clinically. DEN-induced precancerous lesions and hepatocellular carcinoma were dramatically impaired in miR-484 ${ }^{-/}$mice. Mechanistically, ectopic expression of miR-484 initiates tumourigenesis and cell malignant transformation through synergistic activation of the transforming growth factor- $\beta / \mathrm{Gli}$ and nuclear factor- $\mathrm{KB} /$ type I IFN pathways. Specific acetylation of H3K27 is indispensable for basal IFN-induced continuous transcription of miR-484 and cell transformation. Convincingly, formation of precancerous lesions were significantly attenuated in both $\operatorname{Tgfbr}^{\triangle}{ }^{\mathrm{hep}}$ and Ifnar ${ }^{-1-}$ mice.

Conclusions These findings demonstrate a new protumourigenic axis involving type I IFN-microRNA signalling, providing a potential therapeutic strategy to manipulate or reverse liver precancerous lesions and tumourigenesis.

\section{INTRODUCTION}

Precancerous lesions are clinically and histologically altered tissue in which cancer is more likely to develop than in its normal counterpart. ${ }^{1}$ Recognition of the disease at the precancerous stage could significantly prevent the incidence of, and reduce the mortality from, the particular cancers. $^{2} 3$ In the past few years, precancerous lesions have been observed in a wide variety of tumours, including but not limited to colorectal,

\section{Significance of this study}

What is already known on this subject?

- Precancerous lesions are critical to human and mouse hepatocarcinogenesis.

- Therapeutic dose of type I IFN can prevent the progression of hepatocellular carcinoma.

- Blockage of chronic type I IFN signalling could control persistent infections.

- SAMD9L facilitates homotypic fusion of endosomes.

\section{What are the new findings?}

- The critical microRNA cluster of liver precancerous lesion was characterised and the dynamic expression of miR-484 identified as a driving factor for precancerous formation.

- miR-484 can induce hepatocellular malignant transformation in vitro and in vivo. The deletion of miR-484 in miR-484 $4^{-1-}$ mice impairs the formation of precancerous lesions and tumourigenesis.

- Transforming growth factor- $\beta$ and type I IFN pathways are indispensable for the oncogenic role of miR-484. The formation of precancerous lesions was significantly attenuated in both Tgfbr2 $\triangle$ hep and Ifnar1 ${ }^{-l-}$ mice.

- Chromatin modification-involved miRNAs induction may act as the initiating signal linking type I IFN accumulation to hepatocarcinogenesis.

How might it impact on clinical practice in the foreseeable future?

- Our findings demonstrate a new protumourigenic axis involving type I IFN-microRNA-transforming growth factor- $\beta$ signalling, providing a potential therapeutic strategy to manipulate or reverse liver precancerous lesions and tumourigenesis.

gastric, pancreatic, hepatic, cervical and oral cancer. ${ }^{4-7}$ Besides the activation of DNA damage checkpoint and genomic instability, ${ }^{8-10}$ many studies have revealed the important role of persistent inflammation in the generation of precancerous 
A

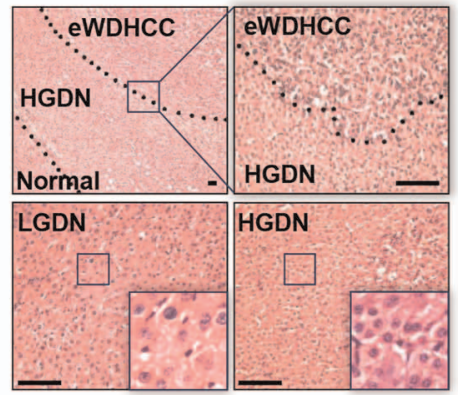

B

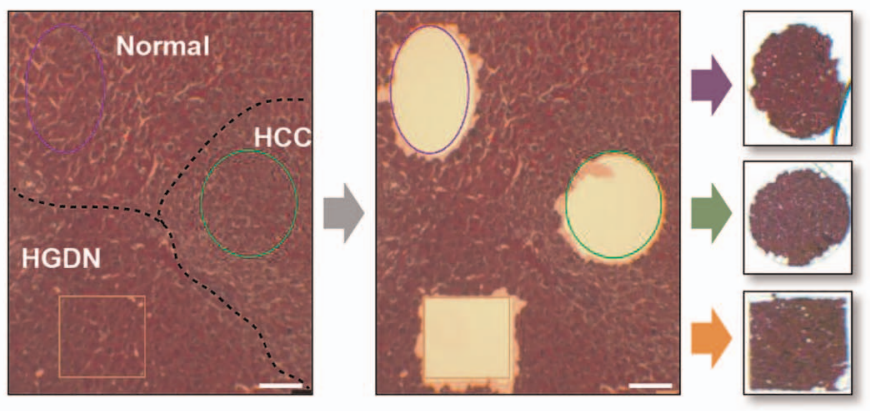

C

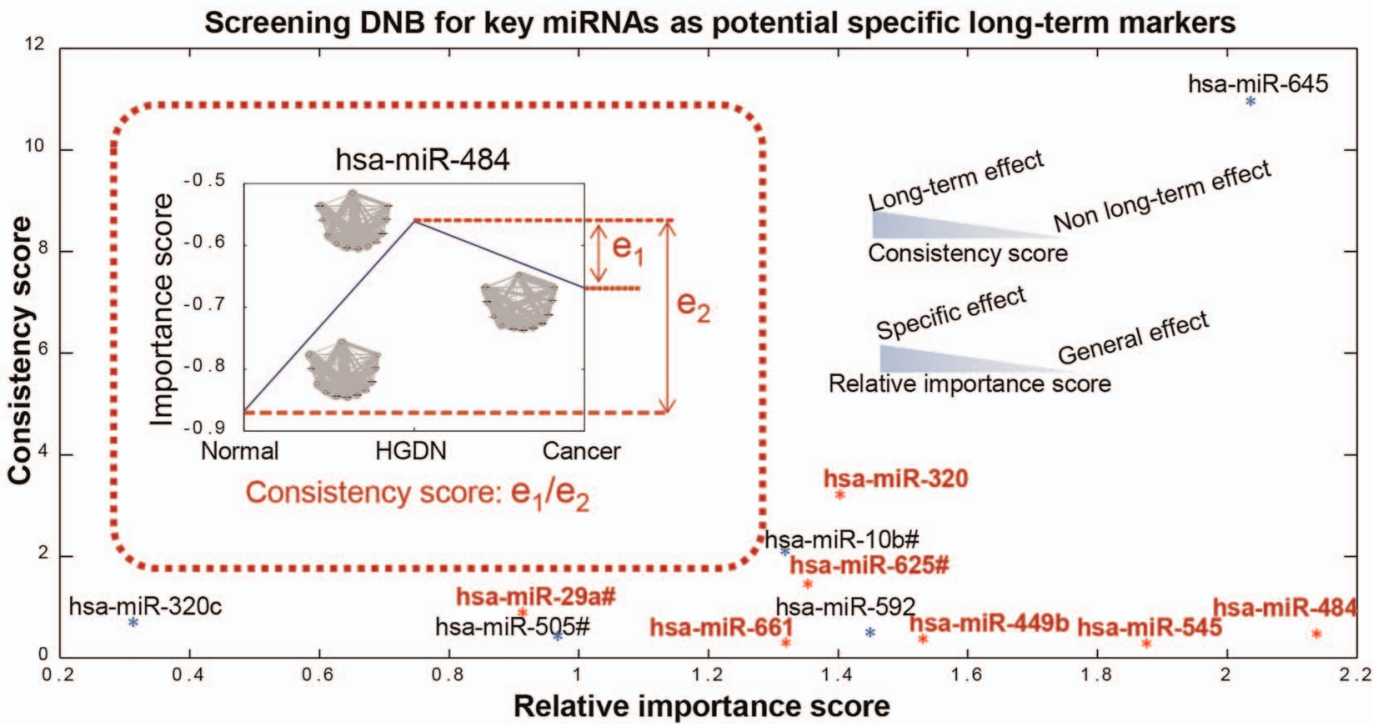

D
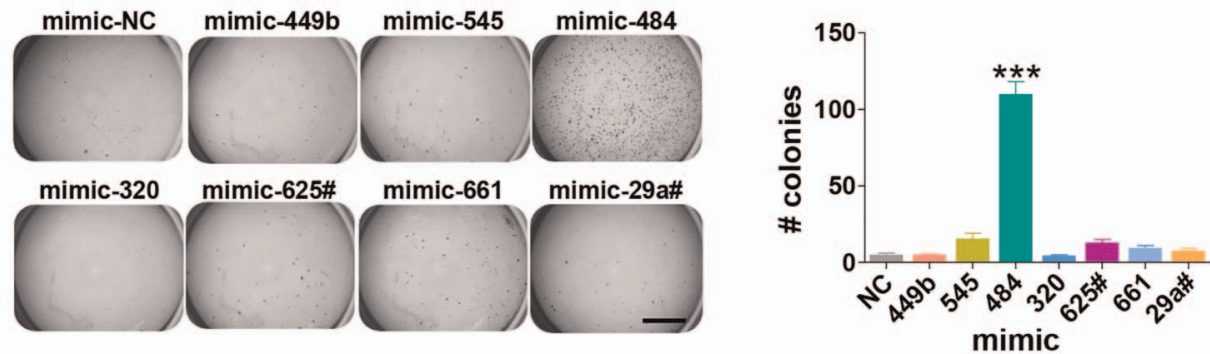

Figure 1 Identification of potential high grade dysplastic nodule (HGDN)-driven miRNAs through dynamic network biomarker (DNB) analysis. (A) H\&E staining of different stage liver tissues in the multistep process of hepatocellular carcinogenesis. Scale bar, $100 \mu \mathrm{m}$. (B) Laser microdissection of paraffin-embedded tissue for normal hepatic tissue, HGDN tissue and hepatocellular carcinoma (HCC) tissue. Scale bar, $100 \mu \mathrm{m}$. (C) Key miRNAs selected based on consistency of network importance. The upregulated miRNAs in HGDN were coloured red. Based on the miRNA profiles for normal cell, HGDN cell and cancer cell, respectively, three miRNA coexpression networks can be constructed and the DNB can be detected. DNB contains a group of miRNAs to indicate the HGDN as a critical point of the pathogen transition from normal to cancer. The key miRNAs are selected from DNB by consistency score and relative importance score, where the two scores are both calculated by the network entropy of DNB miRNAs on the miRNA coexpression networks with bootstrapping. For an miRNA, its large relative importance score means it shows specific effect on and after a critical time, and its small consistency score indicates it has consistent effect/importance after the critical time. Thus, the highest ranked miRNAs should have potential driver ability on the change of biological network/system, for example, the key role of miR-484 in the transition of diseases. (D) Normal hepatocyte was pretreated with indicated microRNA mimics (100 nM) for $48 \mathrm{~h}$, then soft agar colony assay was performed. Colonies (mean \pm SD) $50 \mu \mathrm{m}$ were counted using a microscope 21 days later. Scale bar, $800 \mathrm{~mm}$. (E) Normal hepatocyte was pretreated with lentivirus containing indicated microRNA, then soft agar colonies was performed. Colonies (mean \pm SD) $50 \mu \mathrm{m}$ were counted using a microscope 21 days later. Scale bar, $800 \mathrm{~mm}$. (F) H\&E staining and in situ hybridisation for miR-484 in human HGDN formalin-fixed paraffin-embedded (FFPE) sections. The HGDN specimens were obtained from HBV-infected patient $(n=17)$, miR-484 was overexpressed in most specimens $(n=15)$ and representative positive specimens are shown. Scale bar, $200 \mu \mathrm{m} .{ }^{* * *} p<0.001$. Student's t test.

lesions. ${ }^{11-14}$ However, the underlying molecular mechanisms for their formation remain unclear.

Hepatocellular carcinoma (HCC) is the main type of liver cancer and the second most common cause of cancer mortality worldwide. $^{15}$ It is well known that multihistopathological processes are involved in hepatocellular carcinogenesis, including the generation of large regenerative nodules (LRN), low grade dysplastic nodules (LGDN), high grade dysplastic nodules (HGDN), early well-differentiated HCC (eWDHCC) and moderately differentiated HCC, of which HGDN belongs to the 


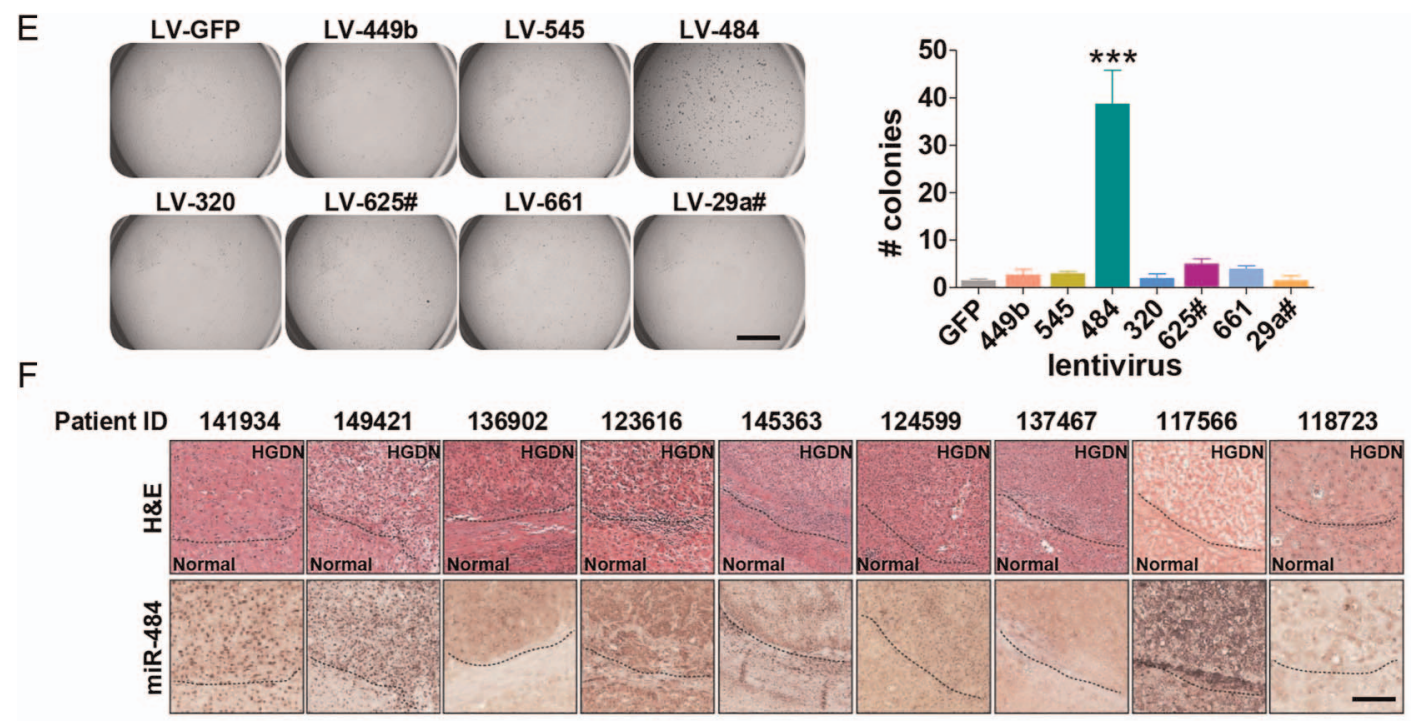

Figure 1 Continued

'borderline malignancy' category, and was defined as the strongest independent predictor of malignant transformation and tumourigenesis in comparison with other non-malignant nodules. ${ }^{16-20}$ According to recent reports, $60 \%-80 \%$ of HGDNs can progress to HCC within 5 years. ${ }^{20-22}$ HGDN often locates in the damaged or cirrhotic liver tissues, and is characterised by a number of features such as morphology, proliferative activity, vascular pattern, DNA content and clonality. ${ }^{12} 162324$

The major risk factor for HCC development is cell deathprovoked chronic inflammation. ${ }^{17}$ Many studies have been dedicated to elucidation of the inflammatory signalling cascades that participate in the initiation of HCC. While several inflammatory-related pathways and cytokines were identified during hepatocarcinogenesis, ${ }^{12}{ }^{25-28}$ little is known about whether chronic inflammation promotes the formation of HGDN, let alone their potential mechanisms and physiological relevance.

Here we determined the role of the type I IFN-miRNA axis for HGDN formation and tumourigenesis in vitro and in vivo, and indicate that chromatin modification-involved induction of

A
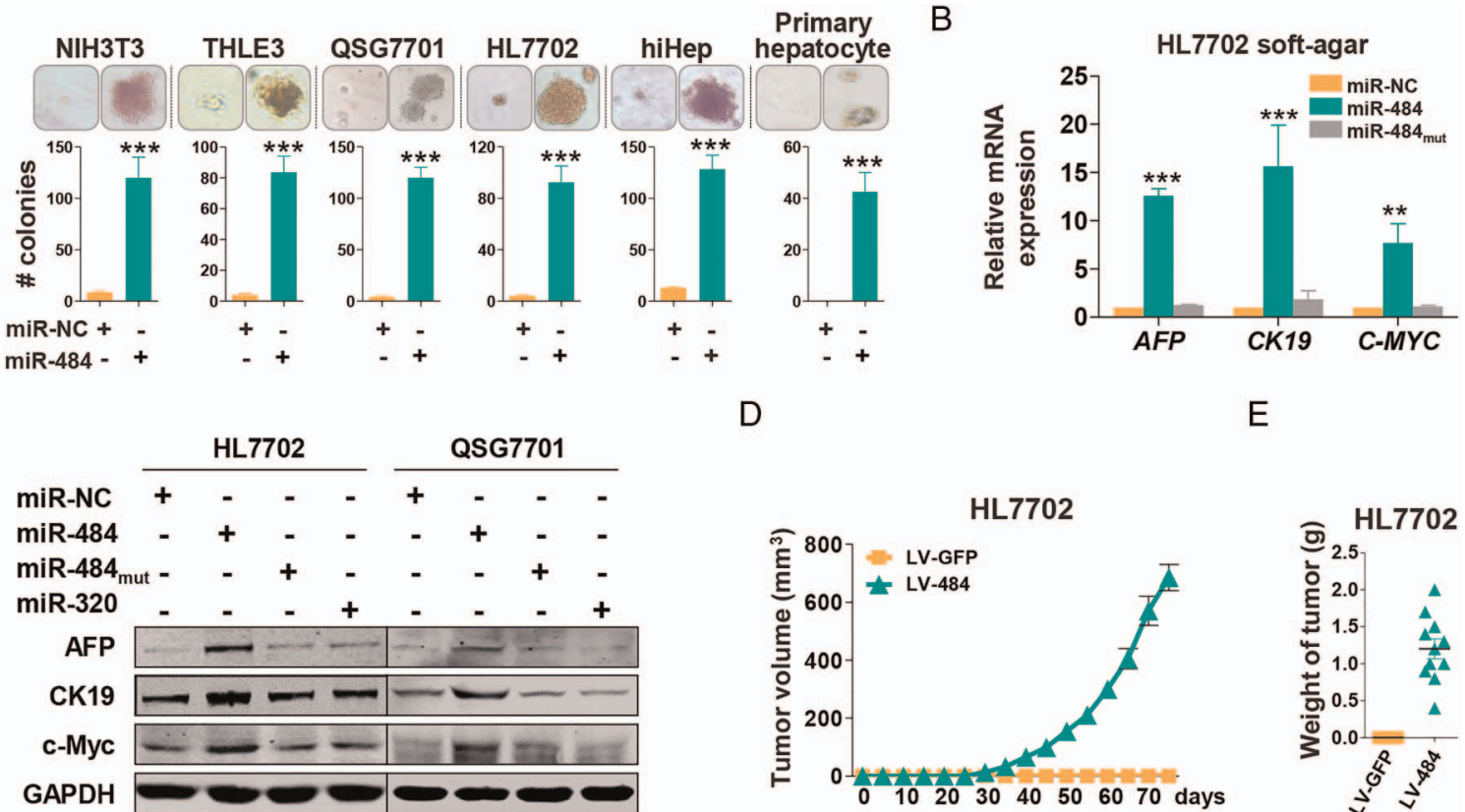

D

E
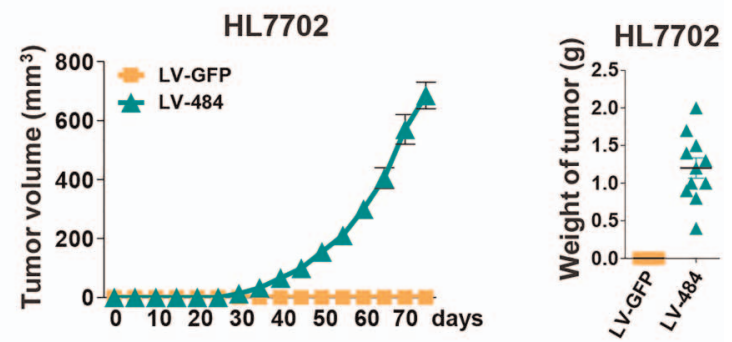

Figure 2 miR-484 enhances hepatocyte malignant transformation. (A) Soft agar colony assay ( $>50 \mu \mathrm{m})$ (mean \pm SD) of indicated cell treated with $100 \mathrm{nM}$ mimic-NC or mimic-484 for $48 \mathrm{~h}$. (B) $\alpha$-fetoprotein (AFP), CK19 and C-MYC mRNA levels (mean \pm SD of three independent experiments) assessed by real-time RT-PCR analysis in soft agar colonies isolated 21 days later. (C) AFP, CK19 and c-Myc protein expression in HL7702 and QSG7701 cells treated with indicated miRNA mimics for $96 \mathrm{~h}$. (D and E) Tumour volume (mean \pm SD) (D) and weight (E) of subcutaneous tumour in NOD.CB17-Prkdcscid/JNju (NOD/SCID)/SCID mice injected with HL7702 cells transfected with lentivirus encoding vehicle or miR-484. (F)

Representative pictures of liver from HL7702 or miHep retrorsine-treated mice. The white arrows indicate tumours. Tumour incidence, multiplicity and size were analysed. (G) H\&E and immunohistochemistry staining in FFPE sections of HL7702 or miHep transplanted liver tissues. Scale bar, $200 \mu \mathrm{m}$. ${ }^{*} p<0.05,{ }^{* *} p<0.01,{ }^{* * *} p<0.001$. 
$\mathrm{F}$
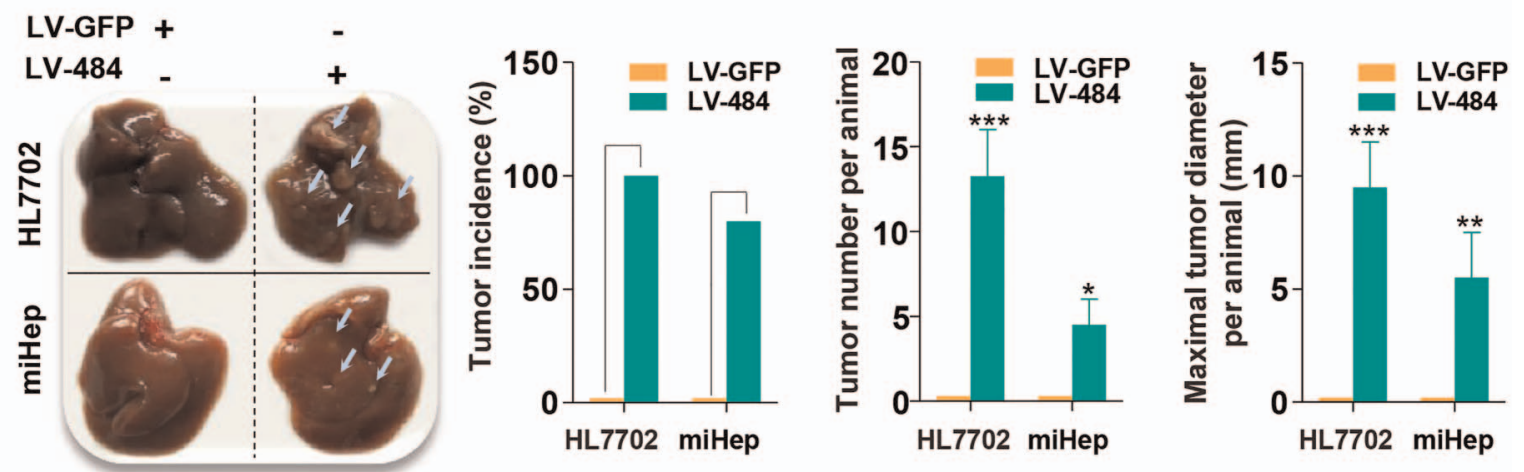

G
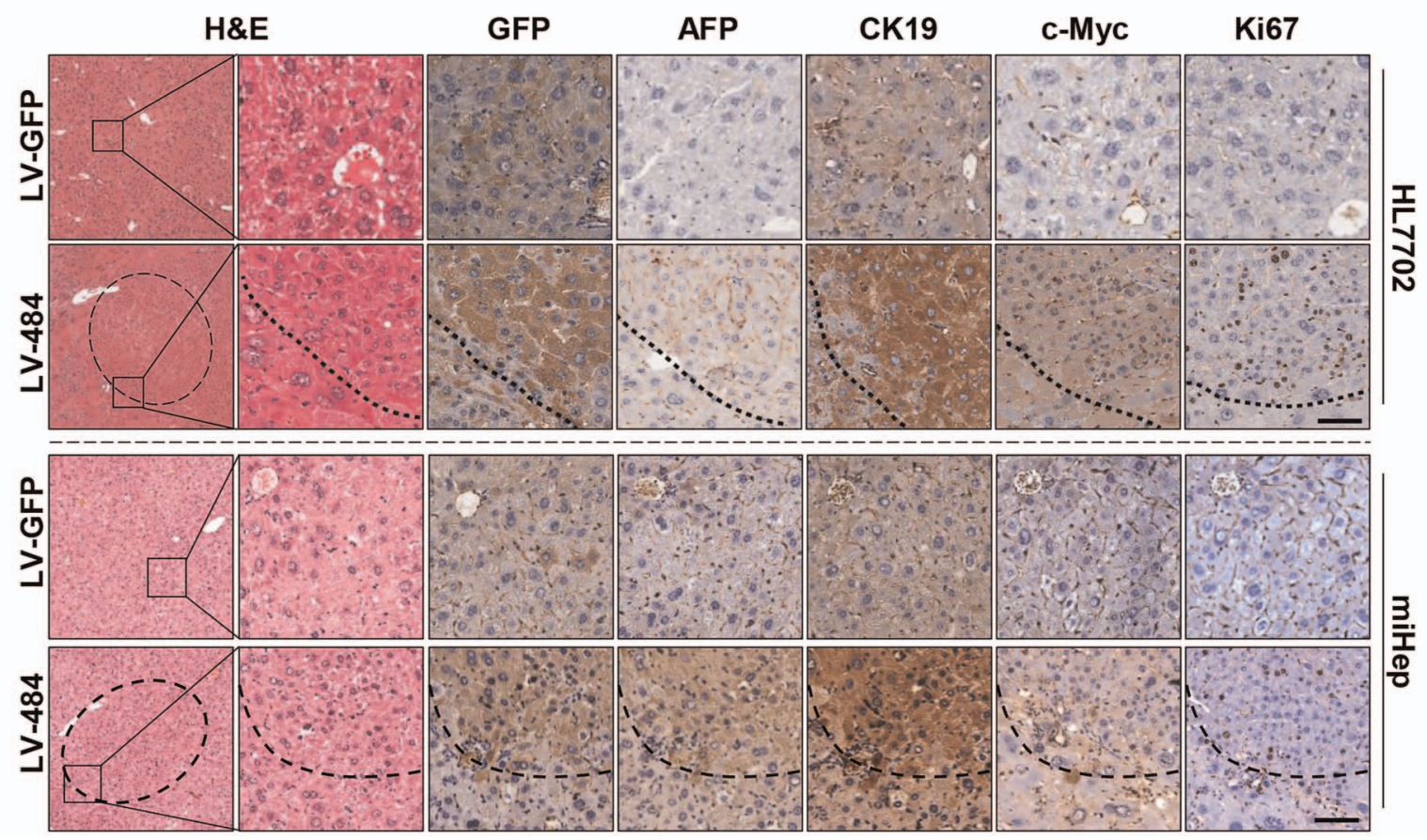

Figure 2 Continued

miRNAs may act as the initiating signal linking type I IFN to hepatocarcinogenesis.

\section{MATERIALS AND METHODS}

\section{Human specimens and their analysis}

All samples used in this study were obtained during liver transplantations or liver resections performed in the Eastern Hepatobiliary Surgery Hospital (Shanghai, China) from 2010 to 2014. These samples were obtained with informed consent according to the Eastern Hepatobiliary Surgery Hospital Research Ethics Committee. Each HGDN specimen was diagnosed consistently by two senior pathologists and the criteria for HGDN reported previously. ${ }^{16} 20$ Detailed experimental description can be found in the online supplementary experimental procedures.

\section{Cell culture}

Cell lines used in this study were THLE-3, NIH/3T3, HL7702, QSG7701, human-induced hepatocytes (hiHeps) and mouse-induced hepatocytes (miHeps). Detailed description of the origin of these cell lines and their culture conditions can be found in the online supplementary experimental procedures.

\section{Mouse experiments}

All experiments of subcutaneous tumour, HCC induction, orthotopic transplantation and therapeutic model are described analytically in the online supplementary experimental procedures.

\section{RNA pull-down assay and RNA immunoprecipitation assay}

The biotin-labelled microRNA pull-down assay and RNA immunoprecipitation (RIP) assay were performed with modifications for using the EZ-Magna RIP Kit (Millipore, 17-701), following the manufacturer's instruction. Detailed experimental description can be found in the online supplementary experimental procedures.

\section{RESULTS}

Characterisation of aberrant microRNA cluster for liver precancerous lesion

The multistep process of hepatocellular carcinogenesis is mirrored by the morphologic classification of lesions observed in liver, including LRN, dysplastic nodules (DN), eWDHCC and HCC (figure 1A). Pathologically, DN is defined as dysplastic nodules of hepatocytes at least $1 \mathrm{~mm}$ in diameter, with dysplasia 
A

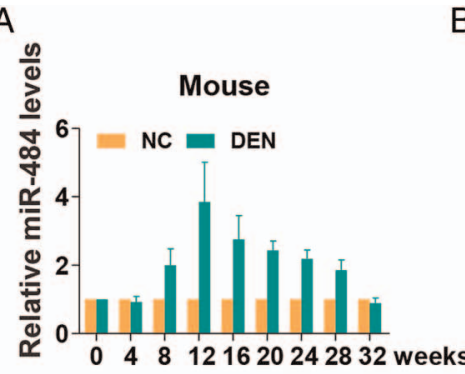

B

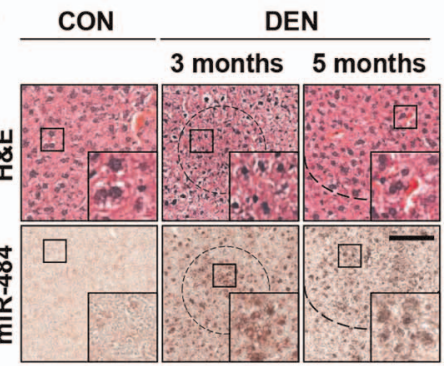

C

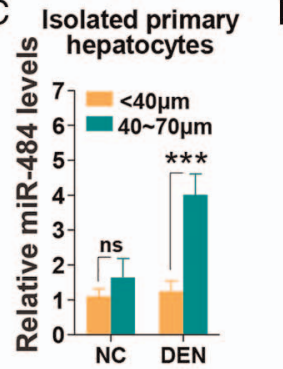

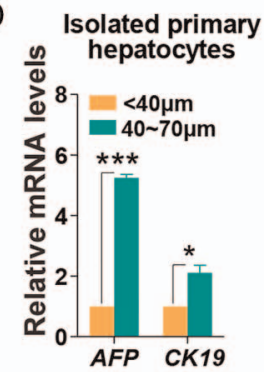

E

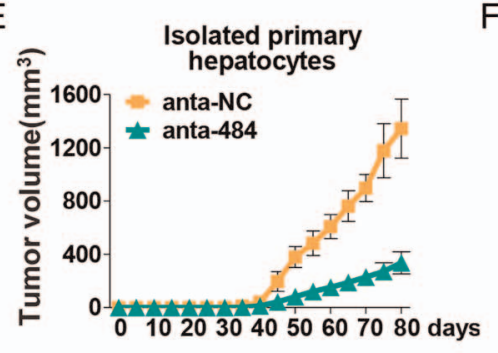

$\mathrm{F}$

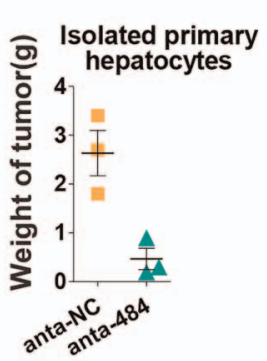

G

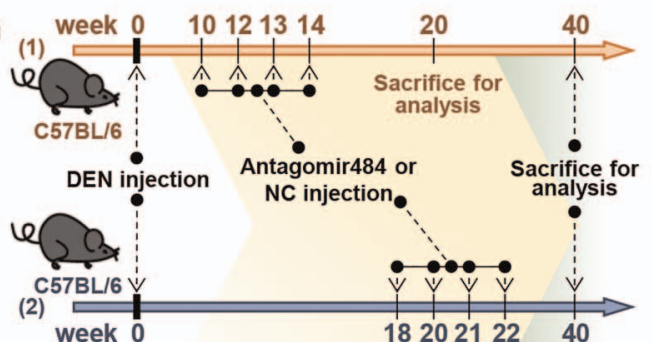

Normal stage Precancerous stage Cancerous stage

I
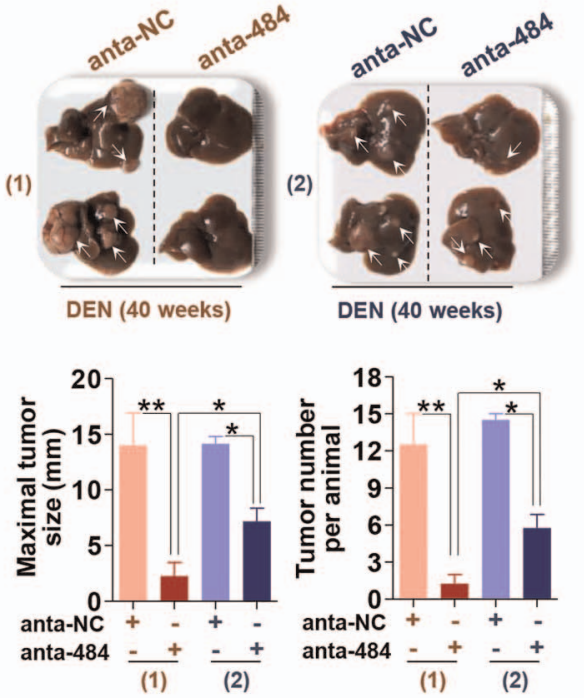

Figure 3 miR-484 is essential for diethylinitrosamine (DEN-induced) hepatocellular carcinoma model. (A) miR-484 expression levels (mean \pm SD) assessed by real-time RT-PCR analysis in liver tissues of DEN-injected mice. (B) Liver sections from 3-month-old or 5-month-old phosphate buffer saline (PBS)-injected or DEN-injected mice were stained with H\&E (top) and in situ hybridisation for miR-484 (bottom). Scale bar, $100 \mu \mathrm{m}$. (C) miR-484 levels (mean \pm SD) assessed by real-time RT-PCR analysis in $<40 \mu \mathrm{m}$ and $40-70 \mu \mathrm{m}$ cells isolated from 5-month-old PBS-injected or DEN-injected mice. (D) AFP and CK19 mRNA levels (mean \pm SD) assessed by real-time RT-PCR analysis in $<40 \mu \mathrm{m}$ and $40-70 \mu \mathrm{m}$ cells isolated from 5-month-old DEN-injected mice. (E and F) Cells of 40-70 $\mu \mathrm{m}$ were treated with $200 \mathrm{nM}$ antagomir-NC or antagomir-484 for $8 \mathrm{~h}$, then injected subcutaneously into NOD/SCID mice with $200 \mathrm{nM}$ antagomir-NC or antagomir-484. Tumours were evaluated 80 days later. Tumour volume (mean \pm SD) (E) and weight (F) were shown. (G) A diagram of antagomir-484 therapeutic delivery experiment. (H) H\&E and immunohistochemistry (IHC) staining in FFPE sections of antagomir-NC and antagomir-484-treated, DEN-treated mice (week 20). Scale bar, $100 \mu \mathrm{m}$. (I) Representative images of livers from indicated mice (top), and tumour size and number were analysed (bottom) (week 40). (J) Identification of conventional miR-484 knockout mice. (K) H\&E and IHC staining of wild-type and miR-484-deficient (miR-484 ${ }^{-l-}$ ) mice that were untreated or treated with DEN for 5 months. Scale bar, $100 \mu \mathrm{m}$. ( $\mathrm{L}$ and $\mathrm{M}$ ) Shown are representative images (L), tumour incidence, size and multiplicity (M) of wild-type and miR-484 ${ }^{-l-}$ mice treated with DEN for 10 months. ${ }^{*} p<0.05,{ }^{* *} p<0.01,{ }^{* * *} p<0.001 ; n s$, not significant.

but without histological criteria of malignancy, and is divided into two subtypes: LGDN and $\mathrm{HGDN}^{21}$ (figure $1 \mathrm{~A}$ ). Accordingly, HGDN has been regarded as a precancerous lesion for hepatocarcinogenesis but not LGDN. ${ }^{20} 21$ To explore the microRNA signature for HGDN formation and hepatocyte malignant transformation, the expression profiles were determined for 754 miRNAs across matched normal hepatocytes,
HGDN and HCC tissues by integration laser capture microdissection method with the TaqMan Low-Density Array (TLDA) assay (figure 1B). Then, a microRNA-based dynamic network biomarker (DNB) analysis was performed to characterise the unique microRNA cluster associated with HGDN phenotypes, revealing 12 miRNAs included in the DNB cluster with extreme deviations in HGDN (see figure 1C and online supplementary 
K

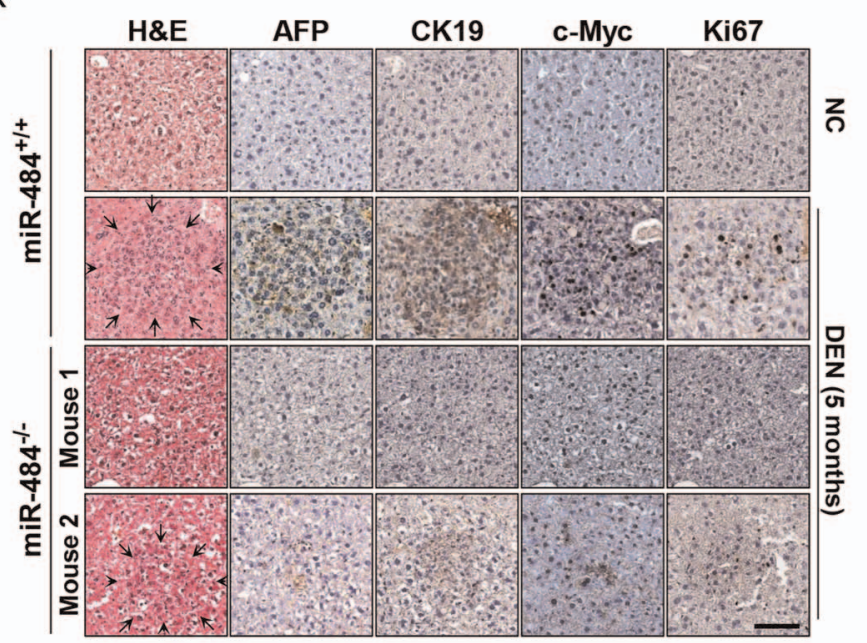

L

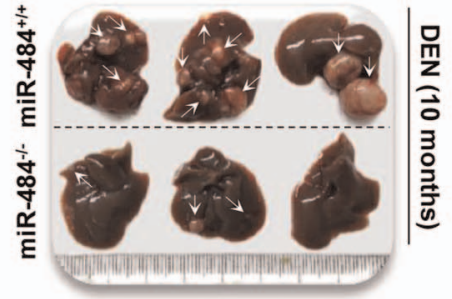

Figure 3 Continued

figure S1A). Among these, seven miRNAs increased in precancerous lesion were regarded as candidate drivers by specific long-term marker analysis in the following studies (figure 1C). Both mimic miRNA transient transfection and lentivirus- mediated stable expression system were applied to verify the capability of those miRNAs for cell transformation through soft agar clonal assays. As shown, only miR-484 exhibits a potential for transformation of an immortalised hepatocyte cell line
A

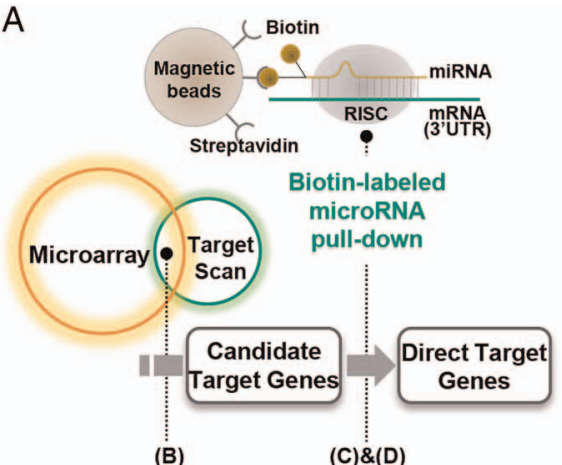

B

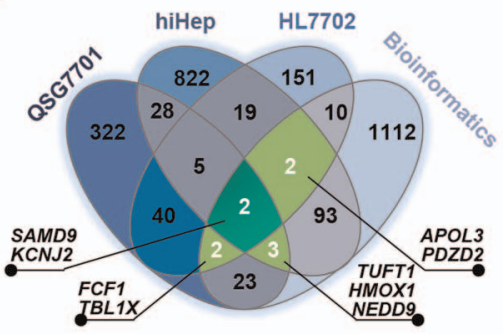

E
C
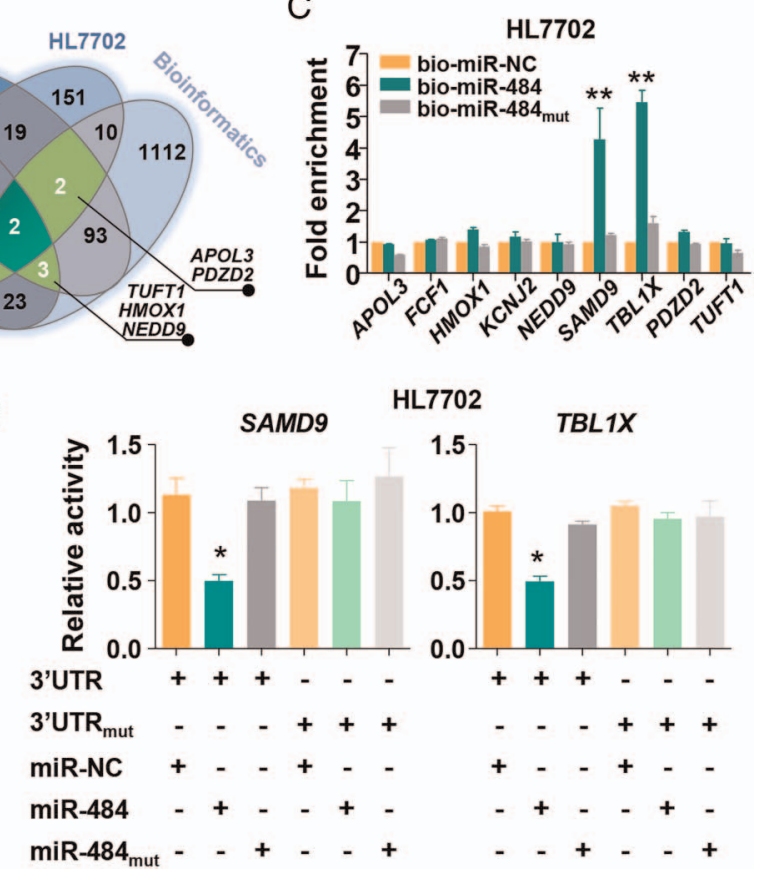

Figure 4 SAMD9 is the bona fide and functional target gene of miR-484. (A) Schematic diagram for target genes discovery. (B) The Venn diagram shows the potential target genes that were shared by TargetScan analysis and decreased mRNA signature of QSG7701, hiHep or HL7702 cells in response to miR-484 treatment. (C) miR-484 can directly bind to 3'UTR of SAMD9 and TBL1X in vivo. HL7702 cells were transfected with biotinylated mimics. After $48 \mathrm{~h}$, cells were harvested for biotin-based pull-down assay. 3'UTR of nine candidate target genes from (B) were analysed by real-time RT-PCR. (D) Representative images of biotin-based pull-down of (C). (E) Luciferase reporter constructs comprising the wild-type or mutant SAMD9 and TBL1X 3'UTR downstream of a luciferase gene were performed. (F and G) Detection of mRNA and protein levels of SAMD9 and TBL1X in HL7702 and QSG7701 cell lines. (H) Real-time PCR analysis of SAMD9L and TBL1X in isolated hepatocytes from miR-484 ${ }^{-l-}$ mice or wild type mice. (I) Immunoblot analysis of SAMD9L and TBL1X in wild-type and miR-484 ${ }^{-1-}$ livers. (J) RNA immunoprecipitation (RIP) experiment was applied using anti-Ago2 with whole-cell extracts of HL7702 cells. The sequences in Ago2 complex were analysed by RT-PCR method. (K) Western blot assays were performed to examine the levels of SAMD9L and TBL1X protein in wild-type MEF or Ago2 knockout MEF cells. (L) The number of colonies was calculated after the induction of si-NC, si-SAMD9 or si-TBL1X. Colonies (mean \pm SD) $50 \mu \mathrm{m}$ were counted using a microscope 21 days later. (M) Soft agar colony formation assay of HL7702 cells cotransfected with miRNA mimics or lentivirus encoding indicated miRNA or mRNA. Colonies (mean \pm SD) $50 \mu \mathrm{m}$ were counted using a microscope 21 days later. Scale bar, $1.5 \mathrm{~cm}$. (N) Levels of AFP, CK19 and c-Myc protein determined by western blot. ${ }^{*} p<0.05,{ }^{* *} p<0.01,{ }^{* * *} p<0.001$. 
$\mathrm{F}$

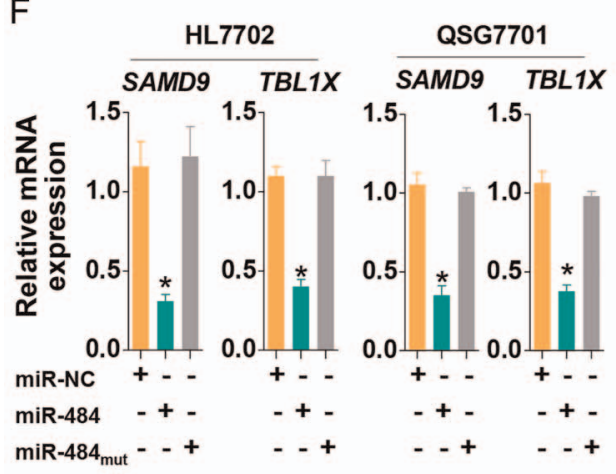

G

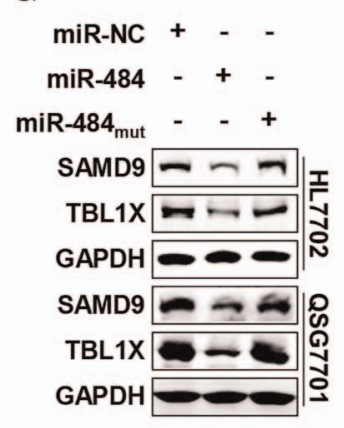

$\mathrm{H}$

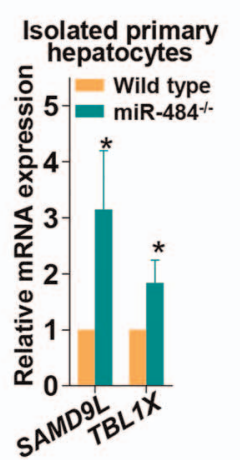

I

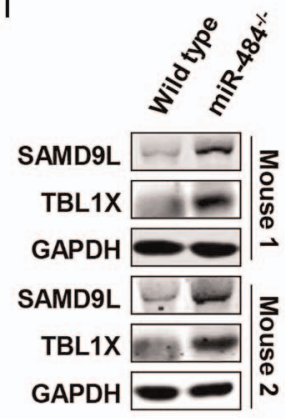

J
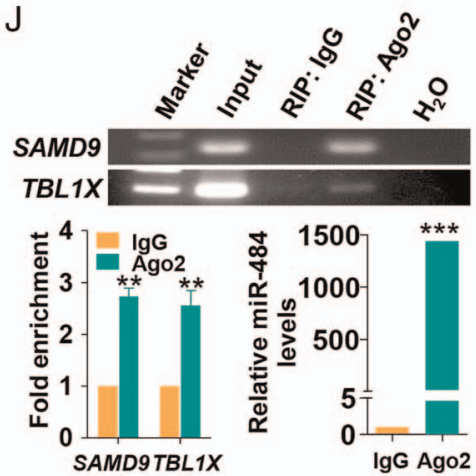

M

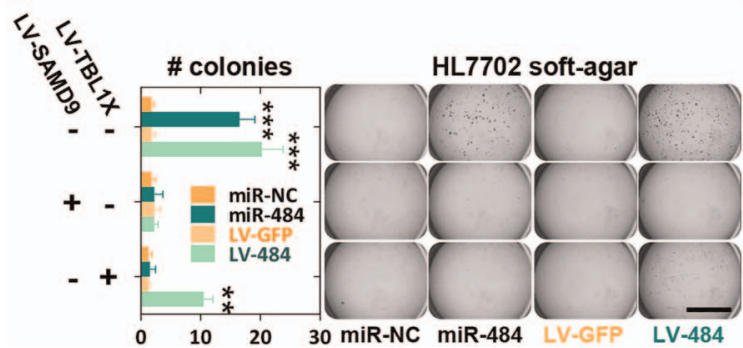

$\mathrm{K}$

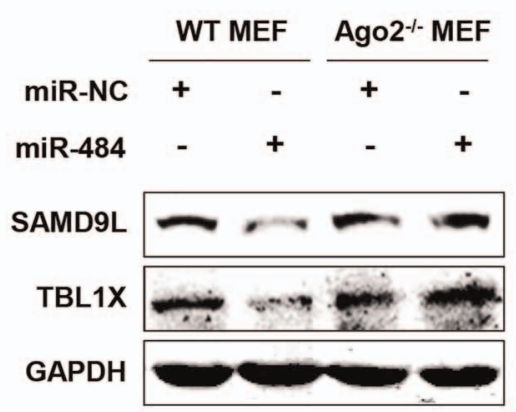

$\mathrm{L}$

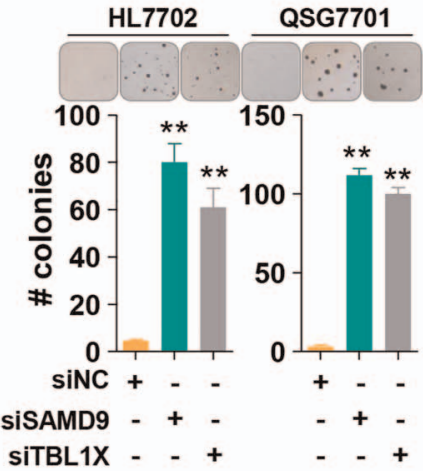

$\mathrm{N}$

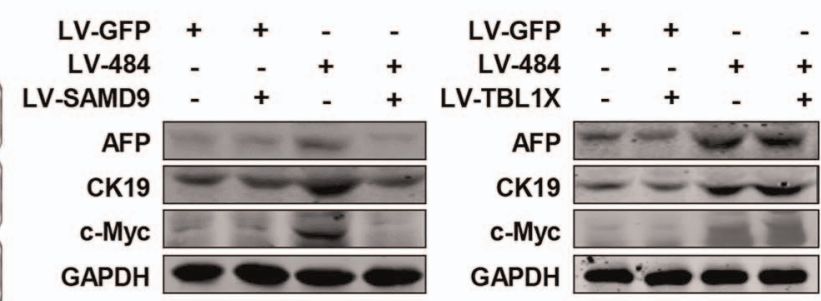

Figure 4 Continued

HL7702 in vitro (see figure 1D,E and supplementary figure S1B,C). Convincingly, in situ hybridisation and real-time RT-PCR assays further showed enrichment of miR-484 in 15 of 17 precancerous HGDN tissues (figure 1F), but not in LGDN (see online supplementary figure S1D), reinforcing the potential role of miR-484 in cell transformation.

\section{miR-484 can induce hepatocellular malignant transformation}

We transiently transfected mimic miR-484, mutant miR-484 or an independent control miR-320 into immortalised normal hepatocytes (HL7702, QSG7701), and transfected mimic miR-484 into NIH3T3, immortalised normal hepatocytes (THLE3), differentiated hepatocyte (hiHep) and primary human hepatocyte, and found similar effects of miR-484 as noted in HL7702 cells (see figure 2A and online supplementary figure S2A-C). Moreover, mRNA and protein expression of two dedifferentiation markers (AFP, CK19) and one malignancy marker (C-MYC) were significantly increased in both isolated soft agar colonies and adherent cultured cells (see figure 2B,C and online supplementary figure $\mathrm{S} 2 \mathrm{D}-\mathrm{G})$. As expected, stable overexpressed miR-484 in normal liver cell line resulted in tumour formation in a murine subcutaneous tumour model (see figure 2D,E and online supplementary figure $\mathrm{S} 3 \mathrm{~A}-\mathrm{C}$ ), and was recovered by antagomir of miR-484 (see online supplementary figure S3D). To further verify the capacity of miR-484 to trigger malignant transformation in vivo, BL/6 mice were first treated with retrorsine, a chemical that permanently inhibits hepatocyte proliferation, ${ }^{29} 30$ and then intrasplenically transplanted with cells stably expressing miR-484 and green fluorescent protein (GFP) (LV-484) or GFP alone (LV-GFP) and then challenged with $\mathrm{CCl}_{4}$ to induce liver injury and compensatory proliferation (see online supplementary figure S3E). The transplantation of miR-484 expressed HL7702 or miHep cells readily formed HCC nodules with strong staining of AFP, CK19, c-Myc and Ki67 and the clustered signals of GFP fluorescence (see figure 2F, G and online supplementary figure S3F-I), which were attenuated after the administration of miR-484 antagomir (see online supplementary figure S3J,K). These in vivo experiments strongly suggest that miR-484 is sufficient to trigger hepatocellular malignant transformation and HCC development in mice.

miR-484 deletion impairs the formation of precancerous lesion and tumourigenesis

To further evaluate whether miR-484 is necessary for HCC development, the expression level of miR-484 was examined in 
A

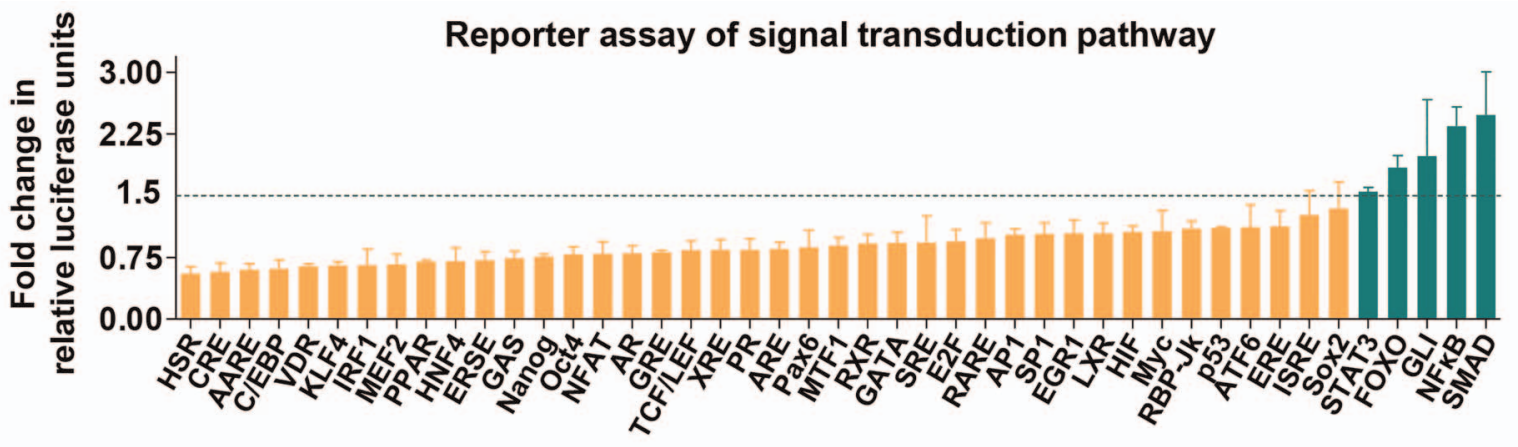

B

C
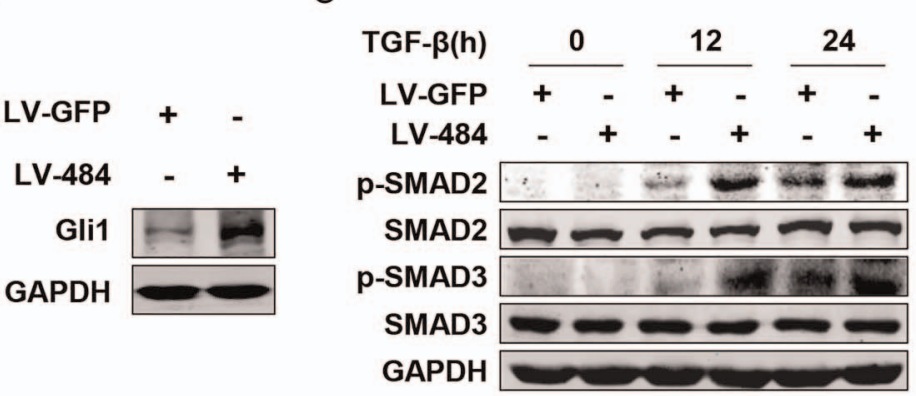

D

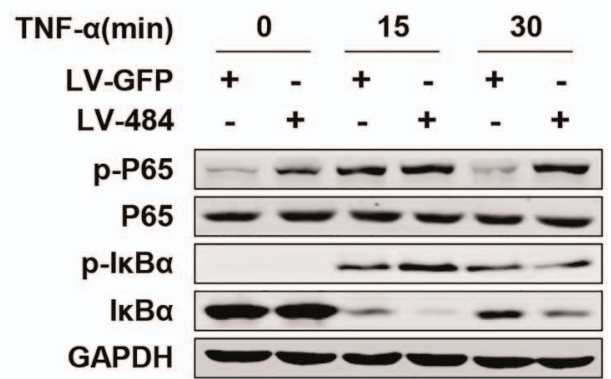

E

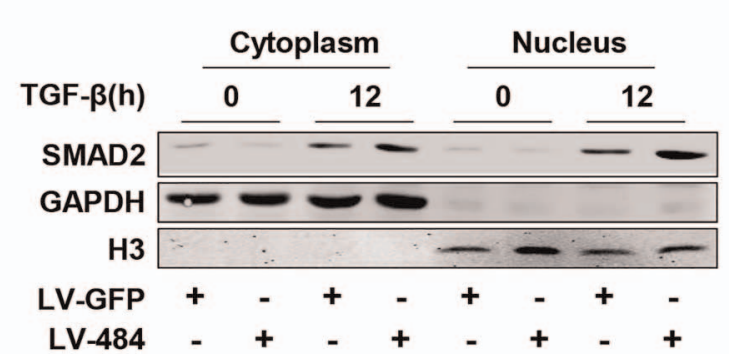

$\mathrm{F}$

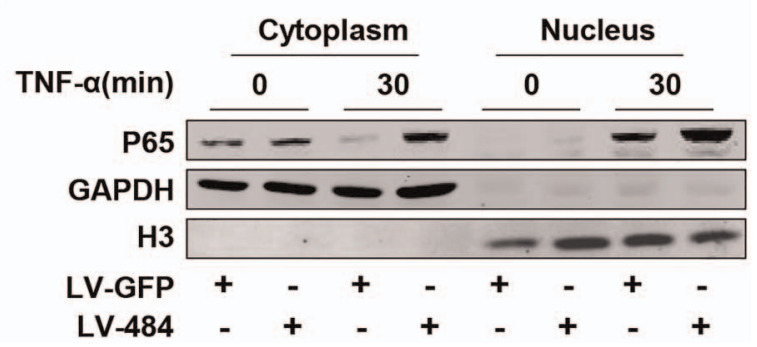

Figure 5 Critical pathways involving miR-484-induced hepatocellular transformation. (A) Cignal Finder 45-Pathway Reporter Assay for cell-based analysis of pathway signalling activity was performed in HL7702 cells transfected with $100 \mathrm{nM}$ mimic-NC or mimic-484 for $48 \mathrm{~h}$. The data are presented as mean $\pm S D$ of three independent experiments. (B-D) The indicated cell line lysates were analysed by western blot with indicated antibodies. (E) Immunoblot detection of cell cytoplasm and nucleus SMAD2, GAPDH and Histone3 of indicated cell lines exposed to transforming growth factor (TGF)- $\beta(10 \mathrm{ng} / \mathrm{mL}$ ) for 0 or $12 \mathrm{~h}$ are shown. (F) Immunoblot detection of cell cytoplasm and nucleus P65, GAPDH and Histone3 of indicated cell lines exposed to tumour necrosis factor (TNF)- $\alpha(20 \mathrm{ng} / \mathrm{mL})$ for 0 or 30 min are shown. (G) The indicated cells were stimulated with TGF- $\beta(10 \mathrm{ng} / \mathrm{mL})$ for $12 \mathrm{~h}$, followed by immunofluorescence assays with SMAD2 and DAPI. The localisations of SMAD2 were detected by confocal laser scanning microscopy as indicated. Scale bar, $100 \mu \mathrm{m}$. (H) The indicated cells were stimulated with TNF- $\alpha(20 \mathrm{ng} / \mathrm{mL})$ for $30 \mathrm{~min}$, followed by immunofluorescence assays with P65 and DAPI. The localisation of SMAD2 was detected by confocal laser scanning microscopy as indicated. Scale bar, $100 \mu \mathrm{m}$. (I) Indicated cell lines were pretreated with indicated inhibitor for $72 \mathrm{~h}$, then soft agar colony assay was performed with the upper medium containing indicated inhibitor for 3 weeks. Colonies (mean \pm SD) $50 \mu \mathrm{m}$ were counted using a microscope 21 days later. $(\mathrm{J}$ and $\mathrm{K}$ ) Tumour volume (mean \pm SD) (J) and weight (K) of subcutaneous tumour in NOD/SCID mice injected with HL7702 cells transduced with lentivirus encoding miR-484, which were treated with indicated inhibitor for $72 \mathrm{~h} .{ }^{* *} \mathrm{p}<0.01$.

the mouse DEN-induced HCC model. ${ }^{12}$ As shown, a gradual increase in miR-484 expression was observed in liver 8 weeks after DEN injection, followed by increased $A f p, c-M y c$ and Ck19 mRNAs 4 weeks later (figure 3A and online supplementary figure S4C). A similar increase in miR-484 expression was also observed in DEN-treated rat liver (see online supplementary figure S4A). More importantly, the specific enrichment of miR-484 was found within precancerous nodules ${ }^{12} 3$ or 5 months after DEN administration (figure 3B). Accordingly, we further enriched the majority of hepatocytes in nodule through 70 and $40 \mu \mathrm{m}$ sieves (see online supplementary figure S4D). Besides the previously documented seven upregulated genes in nodules $^{12}$ (see online supplementary figure S4E), miR-484, Ck19 and $c-M y c$ mRNAs were dramatically increased in these aggregated cells, which implies that the majority of miR-484-transformed hepatocytes consisted of precancerous nodules that can be enriched in the $40-70 \mu \mathrm{m}$ fraction (see figure 3C,D and online supplementary figure S4E). Then, we performed xenograft experiments (see online supplementary figure S4F) in which $40-70 \mu \mathrm{m}$ cell nodules were injected subcutaneously into NOD/SCID mice after antagomir-484 or antagomir-NC treatment, and found that blockage of miR-484 suppressed tumour volume and weight (see figure 3E,F and online supplementary figure S4G-I). To better evaluate the necessity of miR-484 for the formation of precancerous lesions and tumourigenesis, two intervention strategies were designed in which antagomir-484 was administered four times to DEN-treated mice before or after the formation of HGDN 
G
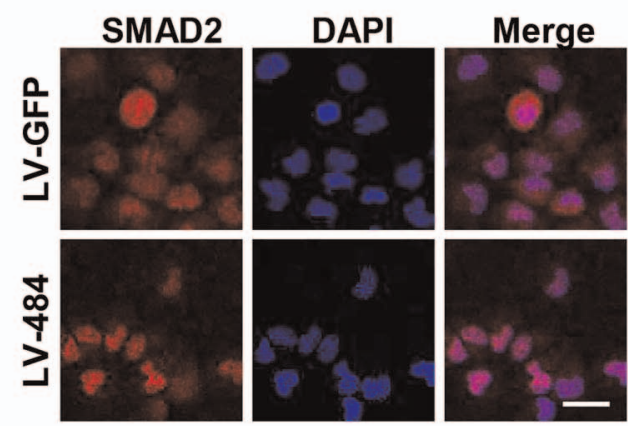

$\mathrm{H}$

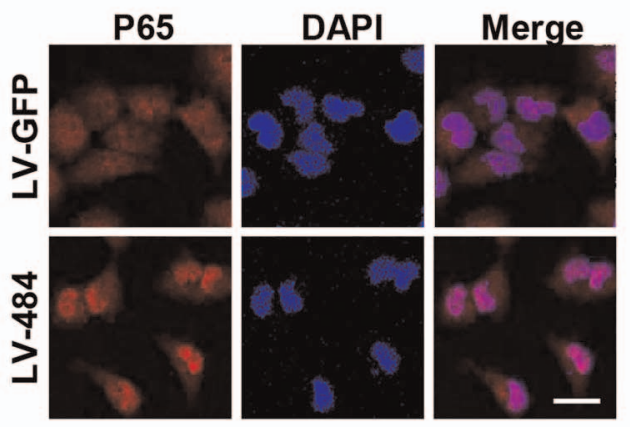

K

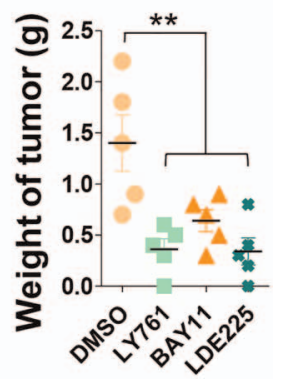

Figure 5 Continued

(figure 3G). As compared with the post-HGDN treated group, injection of antagomir-484 before HGDN formation resulted in the complete elimination of HGDNs (figure $3 \mathrm{H}$ ) and dramatically decreased tumour size or numbers (see figure 3I and online supplementary figure S4J,K). In line with the results obtained from xenograft experiments (figure 3E,F), the post-HGDN treated group showed that blockage of miR-484 cannot completely prevent tumourigenesis (figure 3I). To clearly delineate whether depletion of miR-484 would prevent liver tumourigenicity naturally, conventional miR-484 knockout mice (see figure $3 \mathrm{~J}$ and online supplementary figure $\mathrm{S} 4 \mathrm{~N}$ ) were generated and treated with DEN. Convincingly, DEN-induced premalignant lesions and HCC nodules were significantly ameliorated in miR-484 ${ }^{-1-}$ mice (see figure $3 \mathrm{~K}-\mathrm{M}$ and supplementary figure S4L,M). Taken together, these results reinforce that the induction of miR-484 is an indispensable factor for HCC development in vitro and in vivo.

\section{SAMD9 is the bona fide and functional target gene of miR-484}

In an effort to determine the potential downstream mRNA targets regulated by miR-484 (figure 4A), integrated mRNA expression signatures together with bioinformatics analysis were used to screen for candidate target genes. Genes showing negative correlation with miR-484 expression in at least two cell lines and containing potential $3^{\prime} \mathrm{UTR}$ binding sites were considered as possible candidate target mRNAs (figure $4 \mathrm{~B}$ ). Then, a novel biotin-based microRNA pull-down assay was applied to verify the physical interaction of nine identified candidate target mRNAs, of which two (SAMD9 and TBL1X) were confirmed as the direct targets (see figure 4C,D and online supplementary figure S5A,B). As expected, SAMD9 and TBL1X were dramatically decreased upon exogenous expression of miR-484 specifically (see figure $4 \mathrm{E}-\mathrm{G}$ and online supplementary figure S5C-E), and recovered in miR-484 $4^{-1-}$ mice (see figure $4 \mathrm{H}, \mathrm{I}$ and supplementary figure S5F). In addition, RIP and functional studies confirmed that Ago2 complex is indispensable for miR-484-mediated inhibition of SAMD9 and TBL1X (see figure $4 \mathrm{~J}, \mathrm{~K}$ and online supplementary figure S5G,H). Importantly, both siRNA and lentivirus-mediated gene expression methods verified the necessity of SAMD9 for miR-484-manipulated cell soft agar colony formation and the expression of malignant-related genes except TBL1X, implying that SAMD9 is a functional target of miR-484 (see figure $4 \mathrm{~L}-\mathrm{N}$ and online supplementary figure S5I,J).

Transforming growth factor- $\beta /$ Hedgehog and nuclear factor КB pathways are necessary for miR-484-induced hepatocellular malignant transformation

To elucidate underlying signalling pathway involved in miR-484-triggered hepatocellular malignant transformation, a 45-pathway reporter array was used for cell-based pathway screening, of which five reporter activities (FOXO, GLI, STAT3, nuclear factor (NF) $\kappa \mathrm{B}$ and SMAD) were significantly increased in the presence of miR-484 (figure 5A). After evaluating their upstream manipulators, transforming growth factor (TGF)- $\beta /$ Smad, Hedgehog and NF- $\kappa \mathrm{B}$ pathways were identified as the potential effector pathways influenced by miR-484 (see figure 5B-H and supplemental figure S6A-C). To determine whether these pathways are required for miR-484-induced cell transformation, specific inhibitors (LY2109761 for TGF- $\beta / S m a d, B A Y 11-7085$ for NF- $\mathrm{BB}$ and LDE225 for Hedgehog signalling) were applied separately in the soft agar colony assay in vitro and in tumour formation experiments in vivo. As shown, inhibition of above three pathways dramatically abrogated the miR-484-induced malignant phenotypes, in which administration of the TGF- $\beta$ inhibitor displayed the most significant effects to varying 
A

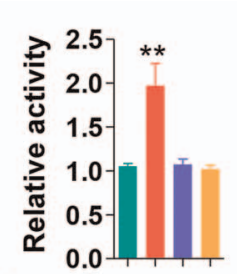

TGF- $\beta$ reporter ++ - -

NF-KB reporter - - + +

siNC $+-+\cdot$

siSAMD9 +++
B

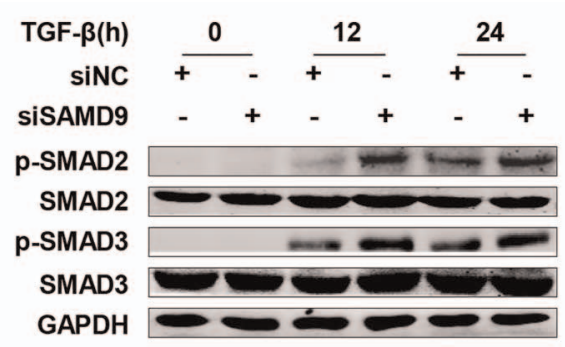

C
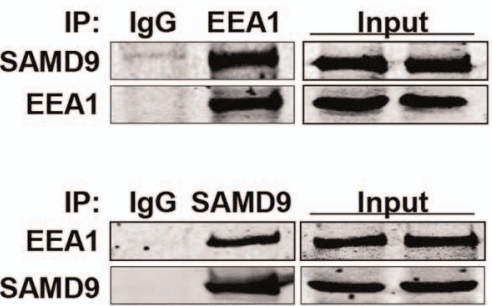

G TGF- $\beta$

$1 \mathrm{~h}$
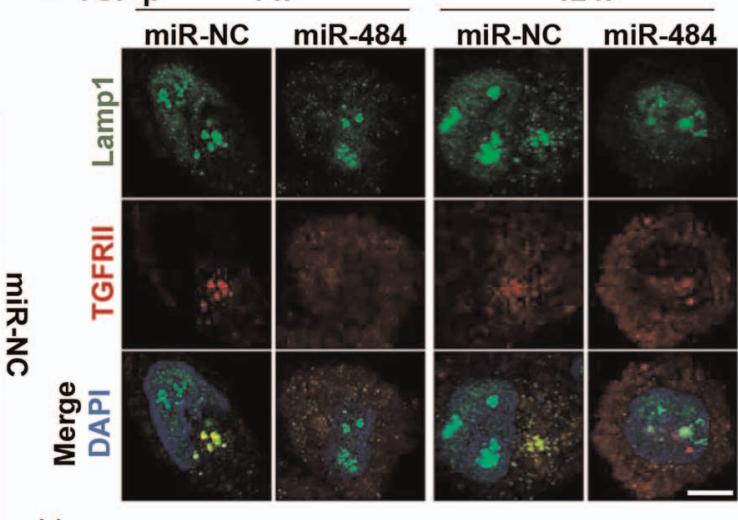

$\mathrm{H}$
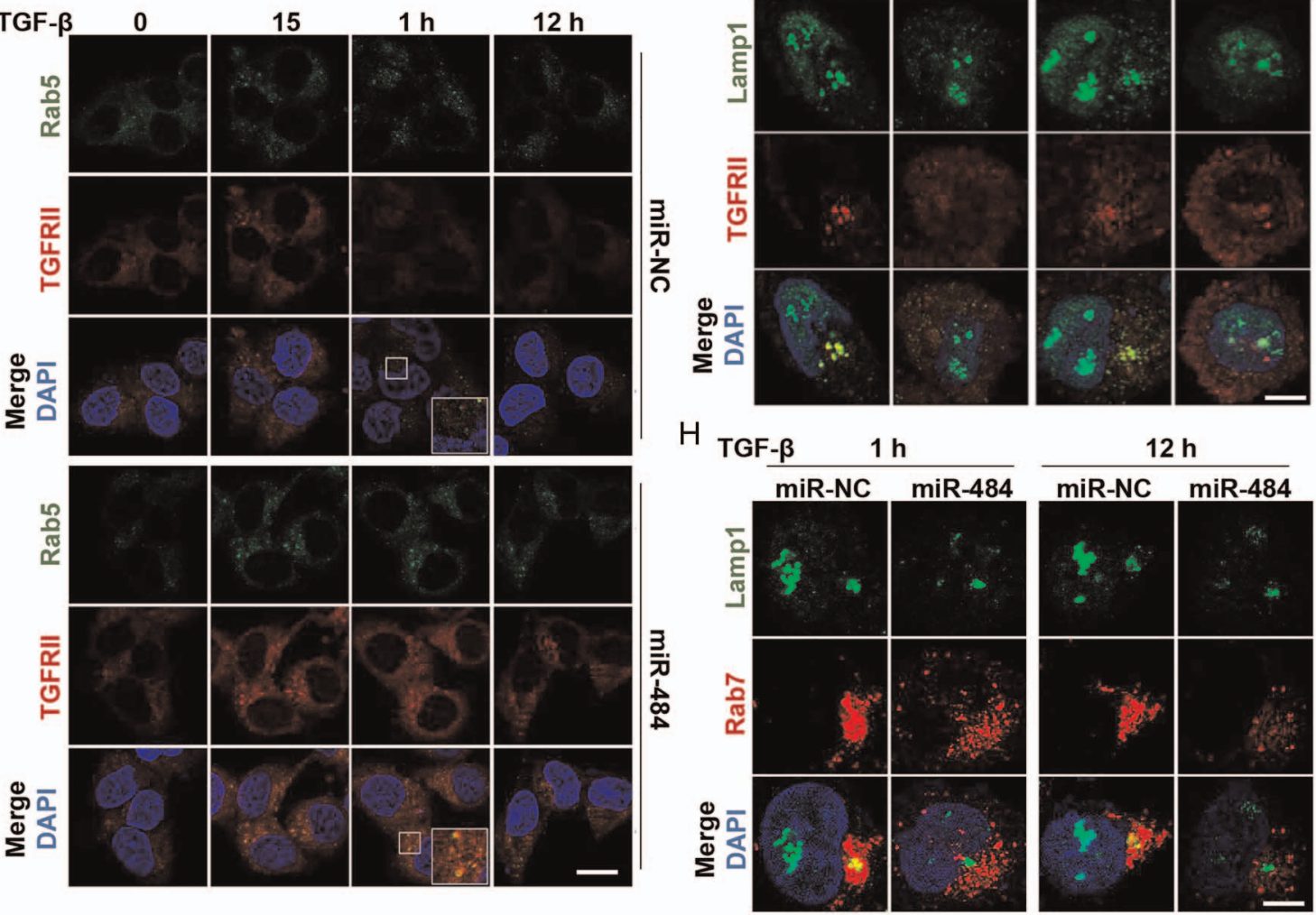

Figure 6 The reduction of SAMD9 upon miR-484 facilitates the recycling of endosomes, prevents lysosome-endosome fusion and enhances TGF- $\beta$ signalling. (A) Luciferase activity (mean \pm SD) of transforming growth factor (TGF)- $\beta$ or nuclear factor- $\mathrm{KB}$ reporter in HL7702 cells transfected with siRNA-NC or siRNA-SAMD9 for $48 \mathrm{~h}$. (B) The indicated cell line lysates were analysed by western blot with indicated antibodies. (C) Whole-cell extracts (input) or immunoprecipitated products using control IgG, anti-EEA1 or anti-SAMD9 of HL7702 cells, followed by immunoblotting with antibodies indicated on the left. (D) The indicated cell lines were exposed to TGF- $\beta(10 \mathrm{ng} / \mathrm{mL})$ for the indicated lengths of time. Cells were stained with the antibodies indicated on the left. The colocalisations of TGFRII and Rab5 were detected by confocal laser scanning microscopy as indicated. Scale bar, $30 \mu \mathrm{m}$. (E and F) Shown were the results of immunoblot detection of indicated protein of the indicated cell lines. (G) The indicated cell lines were exposed to TGF- $\beta$ (10 ng/mL) for indicated lengths of time, then stained with Lamp1 and TGFRII antibodies. The colocalisation was detected by confocal laser scanning microscopy as indicated. Scale bar, $10 \mu \mathrm{m}$. (H) The indicated cell lines were exposed to TGF- $\beta(10 \mathrm{ng} / \mathrm{mL})$ for indicated lengths of time, then stained with Lamp1 and Rab7 antibodies. The colocalisation was detected by confocal laser scanning microscopy as indicated. Scale bar, $10 \mu \mathrm{m}$. (I) H\&E staining and immunohistochemistry for indicated antibodies of TGFBR2 ${ }^{/ F F}$ and TGFBR2 ${ }^{\triangle \mathrm{Shep}}$ mice treated with DEN for 5 months. Scale bar, $100 \mu \mathrm{m}$. (J) The indicated cell lines lysates were analysed by western blot with indicated antibodies. (K) The indicated cell lines were treated with DMSO or LY2109761 for $72 \mathrm{~h}$, then stimulated by TGF- $\beta(10 \mathrm{ng} / \mathrm{mL})$ for $24 \mathrm{~h}$. The detection of Gli1 and GAPDH are shown. ${ }^{* *} p<0.01$.

degrees (see figure 5I-K and online supplementary figure S6IK). Administration of miR-484 also enhanced the expression of IL-6 (see online supplementary figure S6D-E), a key mediator of HCC development, ${ }^{12}$ suggesting that IL- 6 signalling might play a potential role in miR-484-initiated liver tumourigenesis. Since no significant differences were observed in IL-6-targeted p-STAT3 signalling in the presence of miR-484 (see online supplementary figure S6C), further studies are warranted to clarify the physiologic function of miR-484-induced IL-6 in liver dysplasia.
The reduction of SAMD9 facilitates the recycling of endosomes and TGF-receptor relocation

Luciferase reporter assays were applied to clarify the possible

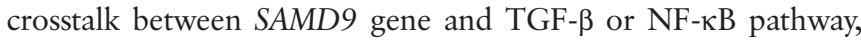
revealing negative regulation of SAMD9 on TGF- $\beta /$ Smad signalling but not the NF- $\mathrm{\kappa B}$ pathway (see figure $6 \mathrm{~A}$ ). As expected, the levels of SMAD2 and SMAD3 phosphorylation were inhibited upon expression of SAMD9 (see figure 6B and online supplementary figure $\mathrm{S7A}$ ). It was reported that $S A M D 9 L$, a paralogous gene of SAMD9, and EEA1 encode crucial 
$E$
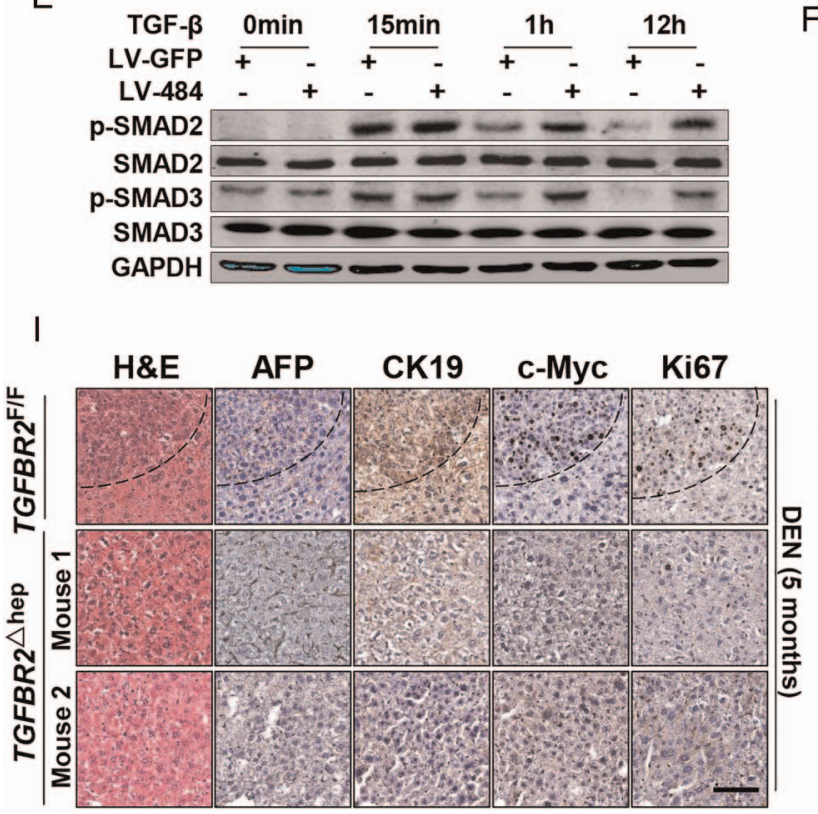

F

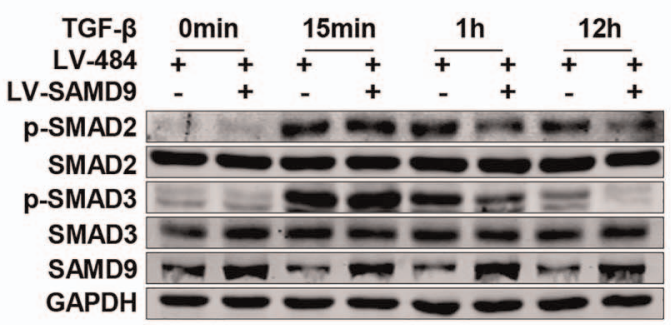

$J$

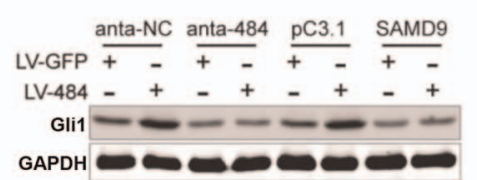

K components of a protein complex that facilitates degradation of PDGFR. ${ }^{31}$ Thus, the question arose whether SAMD9 could exert a similar effect on TGF signalling. Immunoprecipitation and immunofluorescence confocal microscopy revealed proteinprotein interaction between endogenous EEA1 and SAMD9 (see figure $6 \mathrm{C}$ and online supplementary figure $S 7 \mathrm{~B}$ ). Since the internalisation of TGFRI/II is necessary for TGF- $\beta$-mediated signalling, 3233 intercellular distribution of TGFRI/II was examined after TGF- $\beta$ administration, revealing that the colocalisation of TGFRII and Rab5, early endosome marker, was significantly enhanced in the presence of miR-484 (see figure 6D and online supplementary figure S7C for TGFRI and EEA1). Interestingly, SMAD2 and SMAD3 phosphorylation was strongly increased at 15 min followed by a rapid decline in miR-NC cells, while in miR-484 cells it was maintained till $12 \mathrm{~h}$ after TGF- $\beta$ treatment (figure $6 \mathrm{E}, \mathrm{F}$ ). After checking the colocalisation of lysosomeassociated membrane protein 1 (LAMP1, marker for lysosome) and Rab7 (maker for late endosome) or TGFRII, the fusion of lysosome and TGFRII-contained late endosome was found notably reduced in response to miR-484 expression (figure 6G, $\mathrm{H})$, suggesting that the reduction of SAMD9 upon miR-484 can activate TGF- $\beta$ signalling via accelerating TGF receptor recycling and blocking late endosome-lysosome fusion-dependent receptor degradation. Convincingly, hepatocyte-specific TGFBR2 knockout mice further verified a critical role for TGF- $\beta$ signalling in the DEN-induced HCC development model and precancerous lesion formation (see figure 6I and online supplementary figure S7D-F).

It was reported that TGF- $\beta$ can induce the expression of hedgehog $(\mathrm{Hh})$ signalling molecule Gli1 in various cell types in a canonical Hh pathway independent manner. ${ }^{34}$ As shown in figure $6 \mathrm{~J}, \mathrm{~K}$, miR-484 can sensitise cells to TGF- $\beta$-induced Gli expression, and, in contrast, the blockage of TGF- $\beta$ signal completely reversed miR-484-provoked Gli expression. In line with the finding that activation of Gli1 expression in miR-484 cells is not controlled by the Ptch/Smo axis (see online supplementary figure $\mathrm{S} 6 \mathrm{H}$ ), the present results imply that the miR-484-mediated downregulation of SAMD9 is necessary for both TGF- $\beta$ and Hh signalling.

\section{The positive feedback circuit involving type I IFN and} H3K27Ac is required for miR-484 expression

Based on previous observations that transient transfection of miR-484 was sufficient to induce hepatocyte malignant transformation, we hypothesised the existence of a positive feedback loop for consistent activation of miR-484 signalling. As expected, the increased levels of new miR-484 transcripts (pri and pre miR-484) were observed upon mimic miR-484 transient transfection (figure 7A). Similar results were also obtained via luciferase reporter assays (see figure $7 \mathrm{~B}$ and online supplementary figure S8A). Interestingly, an increased level of IFN- $\beta$ was observed in the presence of miR-484 (see figure 7C and online supplementary figure S8B). In addition, a conserved IFN-stimulated response element (ISRE) was found in the upstream promoter region of miR-484, and increased levels of pri, pre and matured miR-484 were observed in several normal hepatocyte cells after low dose IFN- $\beta$ treatment (figure 7D,E); in contrast, no similar changes were found with the expression of miR-320 as control. These results were further confirmed with isolated primary hepatocytes after IFN- $\beta$ administration (figure 7G). Both luciferase reporter assays with wild-type or a mutant binding site in miR-484 promoter and chromatin immunoprecipitation (ChIP) analysis revealed physical binding of STAT1 with the miR-484 promoter region (figure $7 \mathrm{~F}, \mathrm{H}, \mathrm{I}$ ). In comparison with other canonical IFN-stimulated genes (ISGs, such as ISG56, OAS1, PML and TRAIL), transcription of miR-484 could be exclusively induced after stimulation with a wide range of IFN concentration from 0.02 to $2000 \mathrm{U} / \mathrm{mL}$ (see online supplementary figure S8G-I). Consistently, only a low dose of IFN $(2 \mathrm{U} / \mathrm{mL})$ showed the capability of promoting normal hepatocyte malignant transformation (figure $7 \mathrm{~J}$ ) and even more recruitment of STAT1 to the miR-484 promoter (see figure 7I and online supplementary figure S8D), in an miR-484-dependent manner (see figure $7 \mathrm{~K}$ and online supplementary figure S8C,J), which suggests a complex mechanism involved in IFN-modulated miR-484 transcription.

Since database analysis revealed a potential H3K27Ac binding region located upstream of miR-484 promoter (see online supplementary figure S8K), C646, a selective small molecule 
A

HL7702 soft-agar

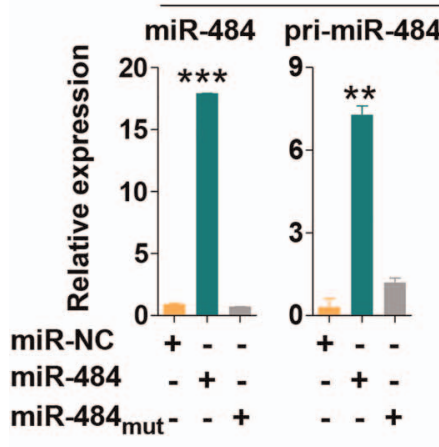

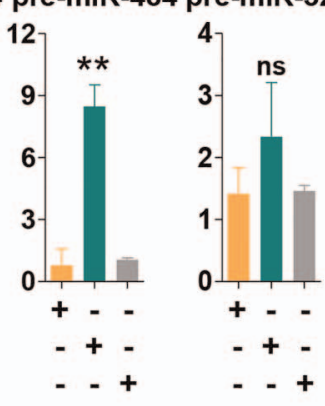

B

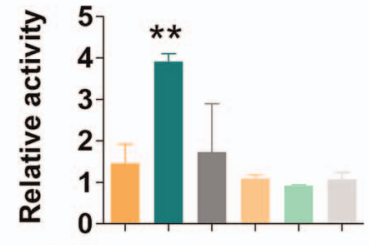

484- promoter $_{\mathrm{wt}}+++$ - - -

484- promoter $_{\text {mut }}$ - - + + +

miR-NC + - +-

$\operatorname{miR}-484-+-++$

miR-484 mut $^{-}-+-+$
C

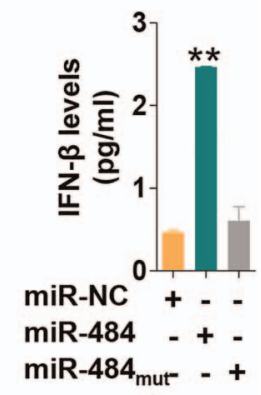

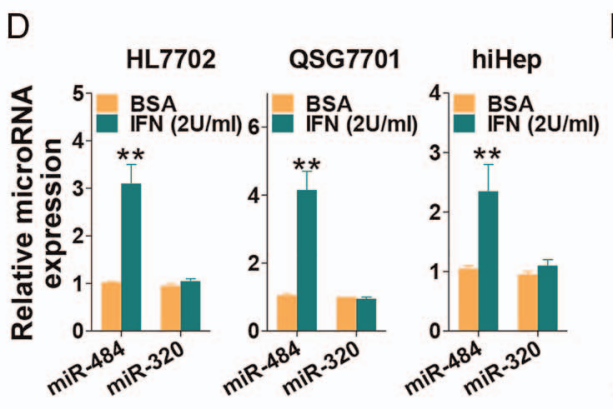

E
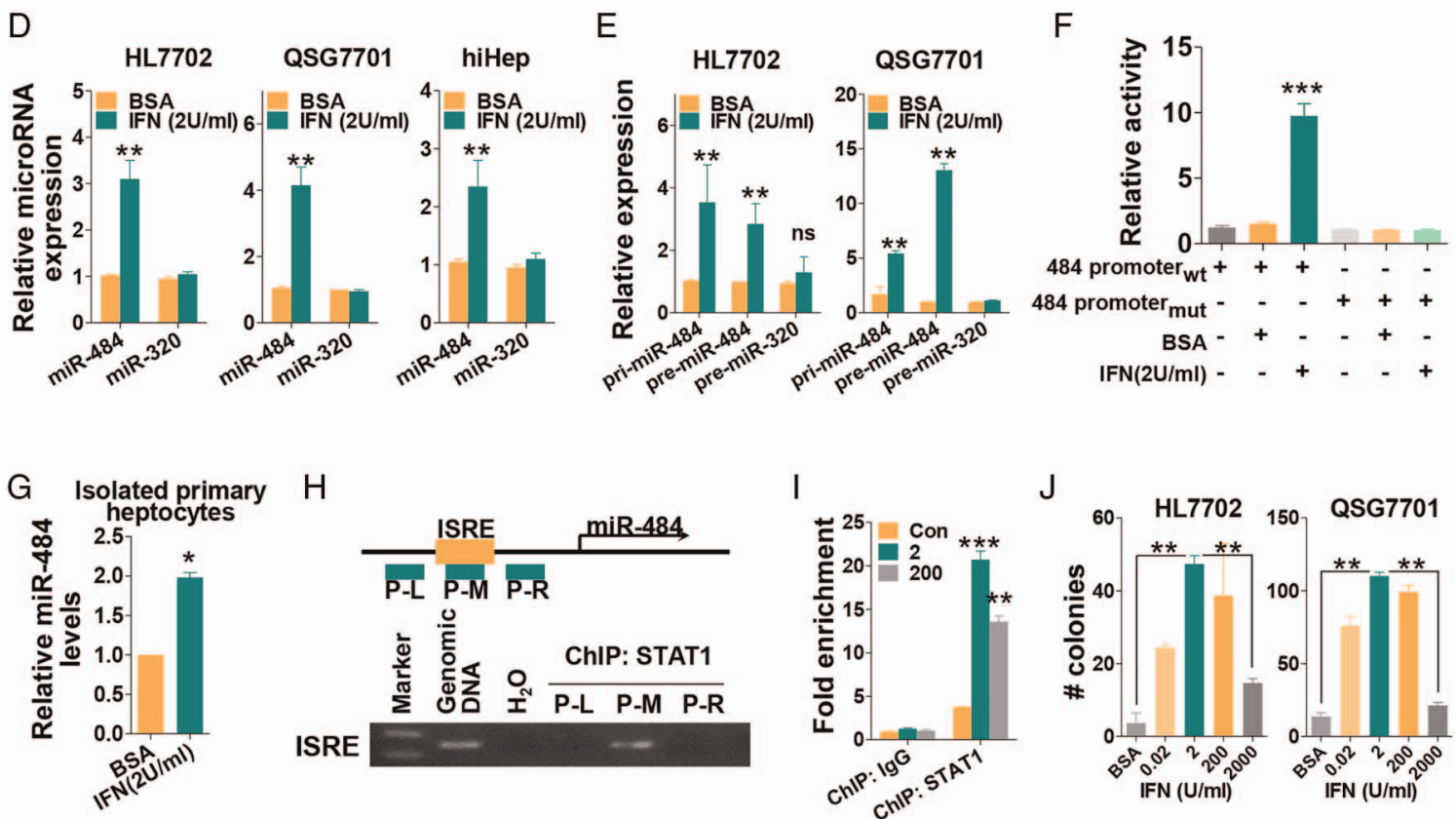

Figure 7 miR-484 is transcriptionally regulated by type I IFN. (A) miR-484 transcript levels (mean \pm SD) assessed by real-time RT-PCR analysis in soft agar colonies isolated 21 days later. (B) Luciferase assays were performed after transduction with $100 \mathrm{nM}$ mimic-NC, wild-type mimic-484 and mutant mimic-484. (C) Levels of IFN- $\beta$ assessed by ELISA in supernatant of indicated cells. (D and E) Transcripts of miR-484 levels (mean \pm SD) were detected by real-time RT-PCR in various cells, with or without stimulation of IFN- $\beta$ for $72 \mathrm{~h}$. (F) Luciferase assays with reporter constructs containing the wild-type or mutant promoter region of miR-484 were performed after stimulation with IFN- $\beta$ for $72 \mathrm{~h}$. (G) Transcripts of miR-484 levels (mean \pm SD) were detected by real-time RT-PCR in isolated primary hepatocytes from mice, with or without stimulation of IFN- $\beta$ for $72 \mathrm{~h}$. (H and I) Chromatin immunoprecipitation from IFN- $\beta$ treated HL7702 cells using STAT1 antibody. (J) Soft agar colony assay (>50 $\mu \mathrm{m})$ (mean \pm SD) of indicated cells treated with different doses of IFN- $\beta$. (K) Soft agar colony assay $(>50 \mu \mathrm{m})$ (mean \pm SD) of HL7702 cotreated with IFN- $\beta$ and $200 \mathrm{nM}$ antagomir. (L) miR-484 levels were assessed by real-time RT-PCR in indicated cells, those cotreated with IFN- $\beta$ and C646 (20 $\mu$ M). (M) Chromatin immunoprecipitation from IFN- $\beta$ and C646 cotreated HL7702 cells using STAT1 antibody. (N) Chromatin immunoprecipitation from IFN- $\beta$ and C646 cotreated HL7702 cells using H3K27Ac antibody. (0) miR-484 levels were assessed by real-time RT-PCR in wild-type and Ifnar1 ${ }^{-1-}$ treated with DEN for indicated time. (P) H\&E staining and in situ hybridisation for miR-484 of wild-type and IFNAR1-deficient $\left(\right.$ Ifnar $\left.1^{-l-}\right)$ mice treated with DEN for 5 months. Scale bar, $100 \mu \mathrm{m}$. ${ }^{*} p<0.05,{ }^{* *} p<0.01,{ }^{* * *} p<0.001$.

inhibitor of histone acetyltransferase p300, ${ }^{35}$ was employed to examine whether histone modification was associated with transcription of miR-484. As shown, administration of C646 specifically attenuated the IFN-induced miR-484 transcription or reporter activity and the binding of STAT1 at the ISRE region (see figure 7L,M and online supplementary figure S8M). Meanwhile, the interaction of $\mathrm{H} 3 \mathrm{~K} 27 \mathrm{Ac}$ with the miR-484 promoter was dissociated upon C646 treatment (see figure $7 \mathrm{~N}$ and online supplementary figure S8L).

Unlike C57/BL6 mice, neither increased miR-484 nor premalignant lesions were observed in Ifnar $1^{-/-}$mice 20 weeks after DEN treatment (figure 7O,P). DEN-induced HCC was also dramatically depressed in the absence of IFNAR1 (see online supplementary figure S9). Collectively, these data demonstrate that miR-484 is transcriptionally regulated by low dose IFN-I with a H3K27Ac-dependent manner, and the activation of type I IFN signalling is necessary for miR-484-induced cell transformation and HCC development.

Physiological relevance of the abnormal miR-484 regulatory circuit with human hepatocellular carcinogenesis

To further verify above findings, the expression levels of miR-484, IFN- $\beta$, SAMD9L and TBL1X were examined by immunohistochemistry in liver tissues of mice 3,4 or 5 months 

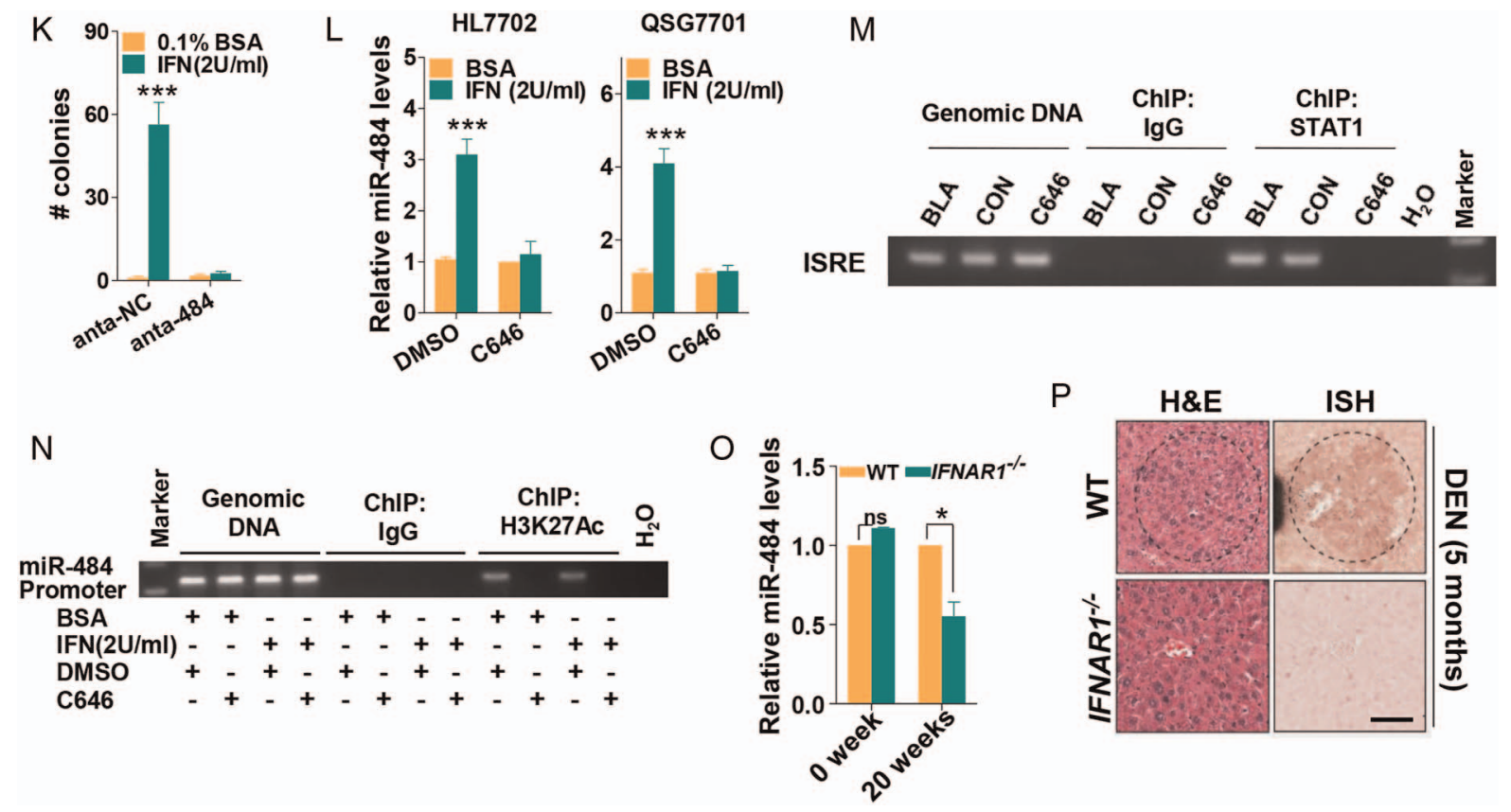

Figure 7 Continued

after DEN administration, revealing the accumulation of miR-484 and IFN- $\beta$ and absence of SAMD9L or TBL1X in premalignant lesion sections in mice (figures $8 \mathrm{~A}, \mathrm{~B}$ and $3 \mathrm{~B}$ ). Further, serial section immunostaining was applied to check the expression of miR-484, IFN- $\beta$, SAMD9 and TBL1X in liver tissues with HGDN lesion from 10 clinical patients (figure $8 \mathrm{C}$ ). More importantly, the positive correlation of serum IFN- $\beta$ concentration with the higher staining of IFN- $\beta$ in HGDN tissues was found in HBsAg positive clinical samples (figure 8D). Strikingly, moderately increased serum level of IFN- $\beta$ was also found in cirrhosis or HCC with chronic hepatitis B virus infection (figure $8 \mathrm{E}$ ). Further, immunostaining of p-SMAD2, p-P65 and Gli1 for DEN samples and clinical samples are applied to prove the molecular scheme (see online supplementary figure S10D,E). Taken together, the present results suggest that chronic HBV infection-induced IFN- $\beta$ accumulation may initiate cell malignant transformation via miR-484-dependent TBL1X or SAMD9 knockdown and the activation of TGF- $\beta / \mathrm{Gli} / \mathrm{NF}-\kappa \mathrm{B}$ pathways by turns (figure $8 \mathrm{~F}$ ).

\section{DISCUSSION}

Inflammation has been identified as the major tumour promoter in various tumours. ${ }^{36} 37$ Being an extraordinarily heterogeneous disease, hepatocyte malignant transformation and HCC are believed to occur through a pathway of increased liver cell turnover, induced by chronic liver injury and regeneration. ${ }^{12} 17183839$ However, the potential contribution of persistent inflammation for HGDN progression has not been elucidated yet. Previous studies have documented that basal enriched IFN-I signalling is associated with disease progression in persistent infections, and blockage of IFN-I signalling reduced immune system tolerance. $^{40-42}$ This is the first study to reveal a slightly and persistently increased level of serum IFN-I in chronic HBV infection, cirrhosis patients and HGDN-containing patients, in parallel with the exclusive staining of IFN in HGDN tissues, implying that persistent activation of IFN may contribute to the formation of dysplastic nodule and tumourigenesis.
In contrast to other ISGs, ${ }^{43}$ the expression of miR-484 is more sensitive to low dose IFN stimulation. Interestingly, the binding of H3K27Ac with miR-484 promoter is independent of IFN stimulation, whereas the interaction of STAT1 and miR-484 is dependent on the presence of H3K27Ac (figure 7L-N), suggesting that $\mathrm{H} 3 \mathrm{~K} 27 \mathrm{Ac}$ is a prerequisite for low dose IFN activity and this chromatin modification conveys selective activation of miR-484 especially under low concentrations of IFN. To further verify the specific function of miR-484, expression of MRAF1 and NDE1 locating nearby miR-484 was measured and no similar enhancement observed in either IFN-stimulated cells or DEN-treated mice (see online supplementary figure S8E,F).

It is well known that cell malignant transformation is the most important process for tumourigenesis, during which accumulated genetic and epigenetic alterations drive normal cells from a precancerous phenotype to a cancerous phenotype. ${ }^{26} 4445$ In recent decades, although several molecular changes that contribute to cell transformation process, including protooncogenes and tumour suppressor genes, were identified, ${ }^{12}{ }^{46-}$

49 most of these conclusions are mainly driven from cell-based experiments, with no in vivo data or clinical sample analysis.

Notably for HCC, despite that precancerous lesion (HGDN) of HCC was identified clinically several years ago, ${ }^{16}{ }^{20}$ the underlying molecular mechanism involving the progression of HGDN and the transition from LGDN to HGDN remains unclear. In addition, since precancerous lesions are generally distributed among normal acini hepatitis or cirrhosis liver tissues, $^{12} 21$ it is hard to collect enough target cells without contamination from other cell types, let alone enough for biomarker screening or functional studies. Here, we verified that the majority of premalignant cells are consistent in HGDN lesion, and knockdown of miR-484 in premalignant cells is sufficient to restrain tumour development. Depletion of miR-484 in mice prevents the formation of precancerous lesions and tumourigenesis. To our knowledge, miR-484 is the first identified miRNA enriched in HGDN tissues. Both bioinformatics and functional studies revealed a major role of miR-484 in HGDN formation instead of the transition from HGDN to 
A

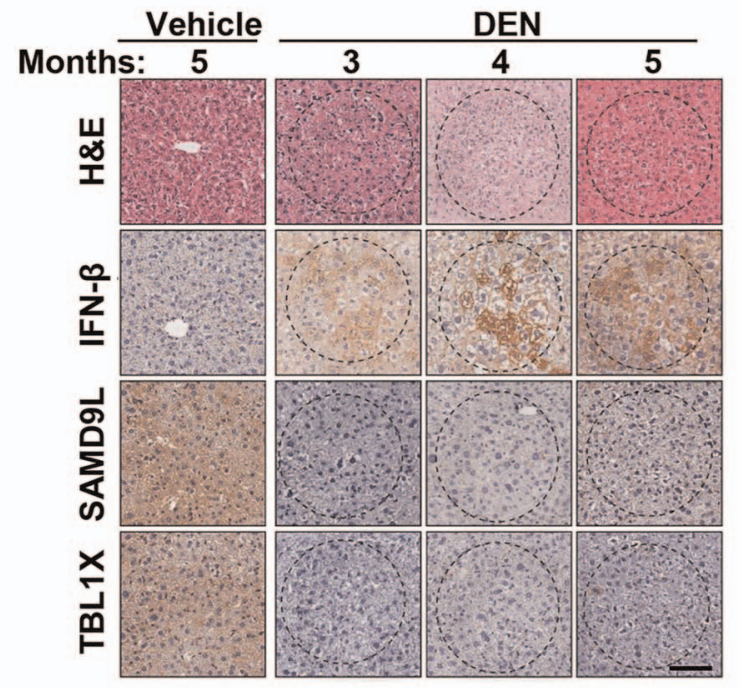

B

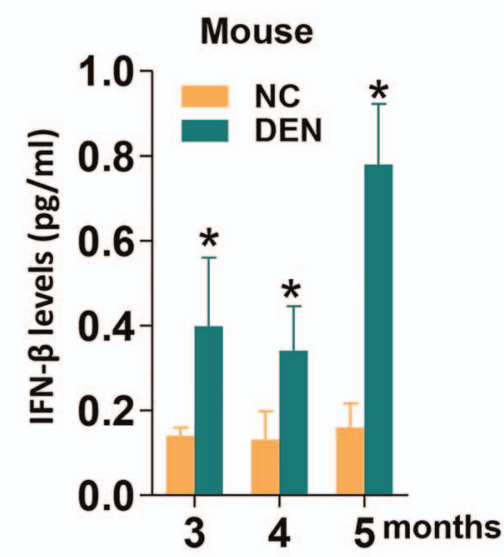

C
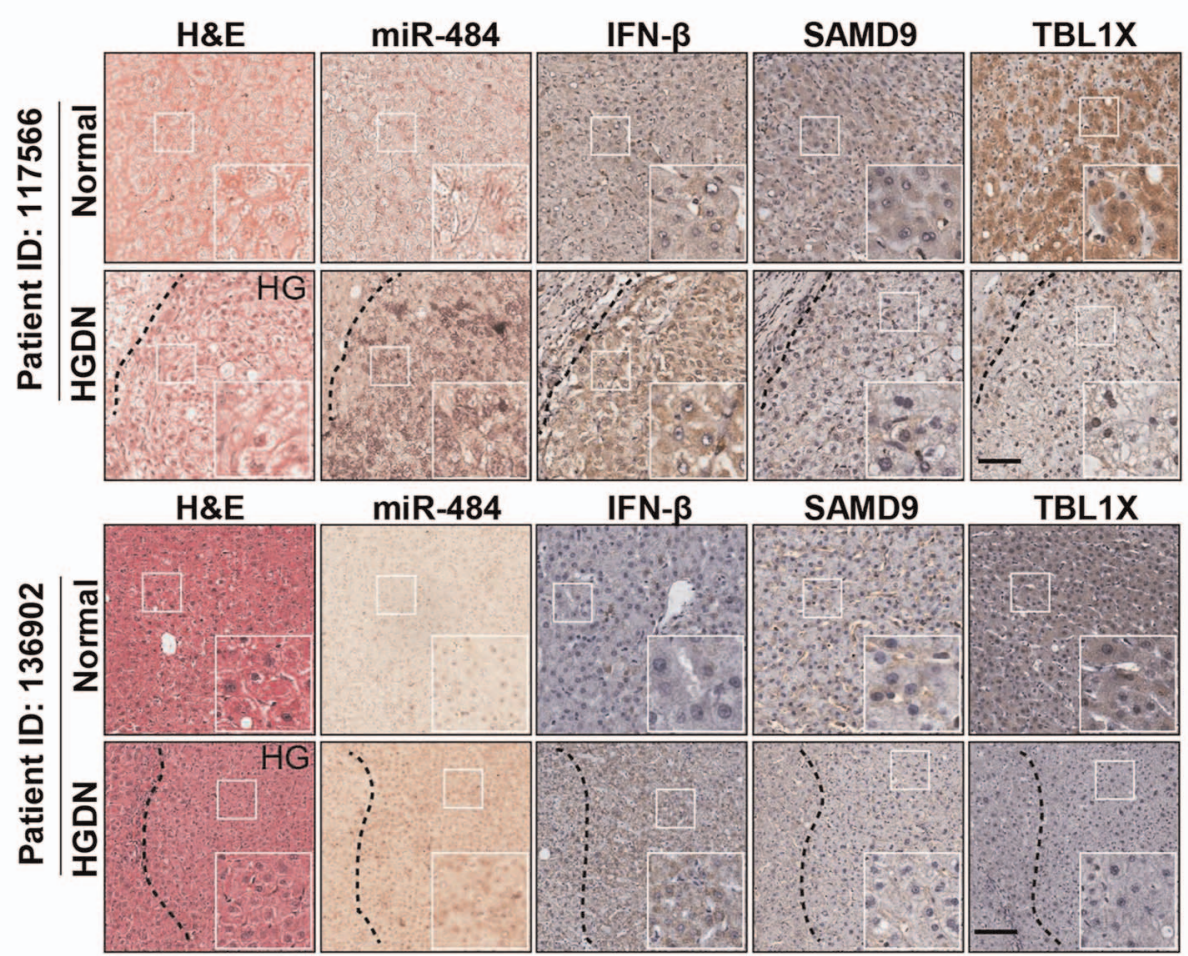

Figure 8 The positive feedback loop of miR-484 involving type I IFN, TBL1X and nuclear factor- $\kappa B$ pathway. (A) Liver sections of DEN mice were subjected to H\&E staining and immunohistochemistry (IHC) with indicated antibodies. Scale bar, $100 \mu \mathrm{m}$. (B) Levels of IFN- $\beta$ assessed by ELISA assay with the serum of C57BL/6 mice treated with DEN or PBS for 3, 4 and 5 months. (C) Representative images of in situ hybridization (ISH) and IHC of 10 high grade dysplastic nodules (HGDN) samples. Scale bar, $100 \mu \mathrm{m}$. (D) Levels of IFN- $\beta$ assessed by ELISA (left) and IHC (right) in the serum or liver sections of the same patient. All serum and sections were obtained from 10 normal samples and three HGDN patients. Scale bar, $100 \mu \mathrm{m}$. (E) Levels of IFN- $\beta$ assessed by ELISA assay with the serum of clinical samples. (F) Schematic representation of the proposed miR-484 circuit during hepatocellular carcinogenesis. ${ }^{*} p<0.05,{ }^{* *} p<0.01,{ }^{* * *} p<0.001$.

HCC, thus reinforcing that HGDN is a unique and unstable stage in hepatocarcinogenesis. Therefore, miR-484 is a practical and effective target to prevent hepatocarcinogenesis through directly reversing premalignant cells in HGDN.

Interestingly, although the inactivation of either IFN-I or TGF- $\beta$ signals shows similar inhibition on the formation of HCC in Ifnar $1^{-/}$or Tgfbr $2^{\text {thep }}$ mice, the dysplastic nodule's number and size are extremely small in Ifnar $1^{-/-}$mice rather than in Tgfbr $2^{\Delta \text { hep }}$ mice. These data indicated that IFN-I signalling maybe potentiate formation of $\mathrm{DN}$ in liver, but is insufficient to drive hepatocarcinogenesis in the absence of TGF- $\beta /$ Gli signalling. Hence, miR-484 may be a key element for the synergetic interaction of IFN-I and TGF- $\beta /$ Gli signalling for hepatocellular malignant transformation.

Together, the present findings elucidated a molecular mechanism responsible for the initiation and maintenance of the hepatocyte-transformed phenotype, which advances our understanding of HGDN's formation and provides a microRNA and IFN-I therapeutic strategy for prevention and intervention of HGDN or HCC. 
D

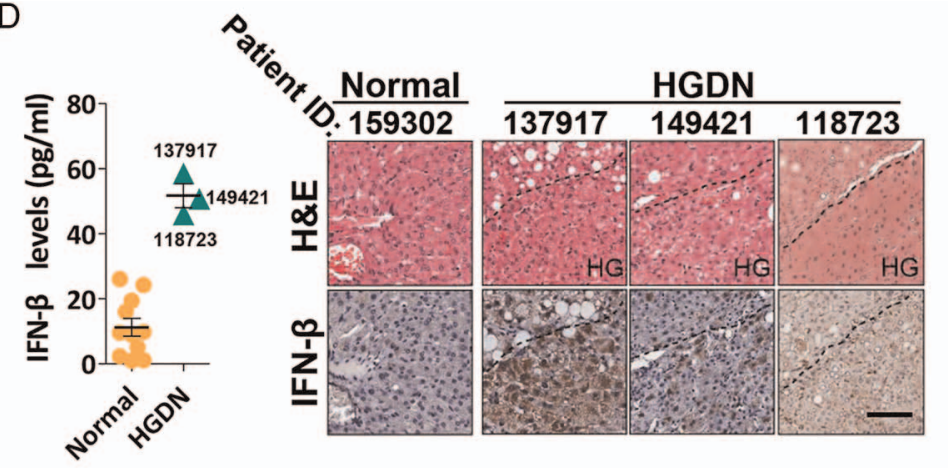

E

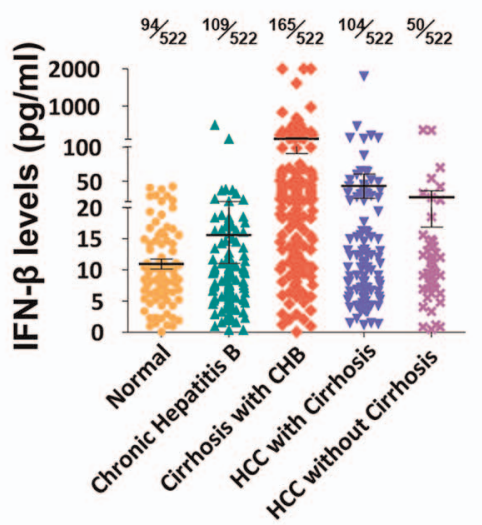

$\mathrm{F}$

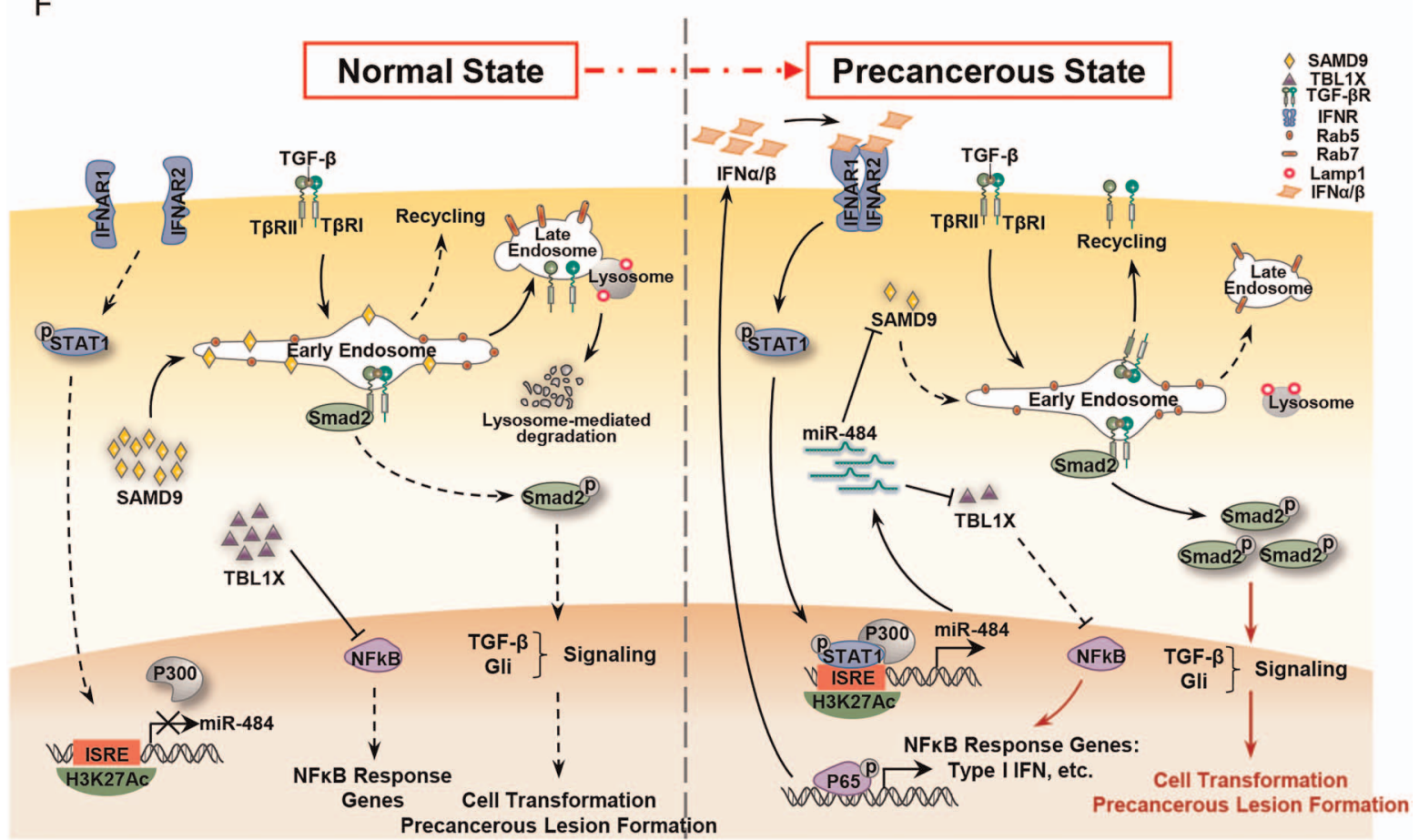

Figure 8 Continued

\section{Author affiliations}

${ }^{1}$ International Co-operation Laboratory on Signal Transduction, Eastern Hepatobiliary Surgery Institute, Second Military Medical University, Shanghai, China

${ }^{2}$ Department of Hepatic Surgery, Eastern Hepatobiliary Surgery Hospital, Shanghai, China

${ }^{3}$ Department of Pathology, Eastern Hepatobiliary Surgery Hospital, Second Military Medical University, Shanghai, China

${ }^{4}$ National Center for Liver Cancer, Shanghai, China

${ }^{5}$ Key Laboratory of Systems Biology, Shanghai Institutes for Biological Sciences, Chinese Academy of Sciences, Shanghai, China

${ }^{6}$ Laboratory of Metabolism, Center for Cancer Research, National Cancer Institute, National Institutes of Health, Bethesda, Maryland, USA

${ }^{7}$ State Key Laboratory for Oncogenes and Related Genes, Cancer Institute of RenJi Hospital, Shanghai JiaoTong University, Shanghai, China

Acknowledgements We are grateful to Professor Lijian Hui for providing hiHep and miHep cells. We would like to thank the members of the International

Co-operation Laboratory on Signal Transduction, especially Dongping Hu, Linna Guo, Dan Cao, Dandan Huang, Shanna Huang and Congli Hu, for excellent technical assistance.

Contributors YCY and XML performed all experiments. XYL, MCW and WMC provided human specimens and pathology identification. TZ, XLC and LNC performed bioinformatics analysis and microarray analysis. XML, JT, FJ, LL, SHT and HLS provided support with experimental techniques. GJL, WTH, GJH, XLC and YQO prepared all samples and clinical data. LC,JGF and HYW designed research and wrote the manuscript.
Funding This work was supported by the state Key project for liver cancer (2012ZX10002-009), the National Research Program of China (2012CB316503, 2012AA02A201), National Natural Science Foundation of China (81221061, $81422032,81272212,81300306,81372674$ and 91529303) and Science Foundation of Shanghai (134119a3700).

Competing interests None declared.

Patient consent Obtained.

Ethics approval Eastern Hepatobiliary Surgery Hospital Research Ethics Committee.

Provenance and peer review Not commissioned; externally peer reviewed.

\section{REFERENCES}

1 Kramer IR, Lucas RB, Pindborg JJ, et al. Definition of leukoplakia and related lesions: an aid to studies on oral precancer. Oral Surg Oral Med Oral Pathol 1978;46:518-39.

2 de Vries AC, van Grieken NC, Looman CW, et al. Gastric cancer risk in patients with premalignant gastric lesions: a nationwide cohort study in the Netherlands. Gastroenterology 2008;134:945-52.

3 Mariotto $A B$, Yabroff KR, Shao $Y$, et al. Projections of the cost of cancer care in the United States: 2010-2020. J Nat/ Cancer Inst 2011;103:117-28.

4 Sandouk F, Al Jerf F, Al-Halabi MH. Precancerous lesions in colorectal cancer. Gastroenterol Res Pract 2013;2013:457901.

5 Genta RM, Rugge M. Gastric precancerous lesions: heading for an international consensus. Gut 1999;45(Suppl 1):15-8. 
6 Costa A, Zanini V. Precancerous lesions of the breast. Nat Clin Pract Oncol 2008:5:700-4.

7 Roncalli M, Terracciano L, Di Tommaso L, et al. Liver precancerous lesions and hepatocellular carcinoma: the histology report. Dig Liver Dis 2011;43(Suppl 4): S361-72.

8 Vogelstein B, Fearon ER, Hamilton SR, et al. Genetic alterations during colorectal-tumor development. N Engl J Med 1988;319:525-32.

9 Hermsen M, Postma C, Baak J, et al. Colorectal adenoma to carcinoma progression follows multiple pathways of chromosomal instability. Gastroenterology 2002;123:1109-19.

10 Wootton SK, Halbert CL, Miller AD. Sheep retrovirus structural protein induces lung tumours. Nature 2005:434:904-7.

11 Taniguchi K, Wu LW, Grivennikov SI, et al. A gp130-Src-YAP module links inflammation to epithelial regeneration. Nature 2015;519:57-62.

12 He G, Dhar D, Nakagawa $H$, et al. Identification of liver cancer progenitors whose malignant progression depends on autocrine IL-6 signaling. Cell 2013;155:384-96.

13 Karin M. Nuclear factor-kappaB in cancer development and progression. Nature 2006;441:431-6.

14 Sun L, Miyoshi H, Origanti S, et al. Type I interferons link viral infection to enhanced epithelial turnover and repair. Cell Host Microbe 2015;17:85-97.

15 Torre LA, Bray F, Siegel RL, et al. Global cancer statistics, 2012. CA Cancer J Clin 2015:65:87-108.

16 Roncalli M, Borzio M, Di Tommaso L. Hepatocellular dysplastic nodules. Hepatol Res 2007;37(Suppl 2):S125-34.

17 Forner A, Llovet JM, Bruix J. Hepatocellular carcinoma. Lancet 2012;379: 1245-55.

18 El-Serag HB. Hepatocellular carcinoma. N Engl J Med 2011;365:1118-27.

19 Kudo M. Multistep human hepatocarcinogenesis: correlation of imaging with pathology. J Gastroenterol 2009;44(Suppl 19):112-18.

20 Borzio M, Fargion S, Borzio F, et al. Impact of large regenerative, low grade and high grade dysplastic nodules in hepatocellular carcinoma development. J Hepatol 2003:39:208-14

21 International Working Party. Terminology of nodular hepatocellular lesions. Hepatology 1995;22:983-93.

22 Kobayashi M, Ikeda K, Hosaka T, et al. Dysplastic nodules frequently develop into hepatocellular carcinoma in patients with chronic viral hepatitis and cirrhosis Cancer 2006;106:636-47.

23 Hunecke D, Spanel R, Länger F, et al. MYC-regulated genes involved in liver cell dysplasia identified in a transgenic model of liver cancer. J Pathol 2012;228:520-33.

24 Jin GZ, Yu WL, Dong H, et al. SUOX is a promising diagnostic and prognostic biomarker for hepatocellular carcinoma. J Hepatol 2013;59:510-17.

25 He G, Yu GY, Temkin V, et al. Hepatocyte IKKbeta/NF-kappaB inhibits tumor promotion and progression by preventing oxidative stress-driven STAT3 activation. Cancer cell 2010;17:286-97.

26 Iliopoulos D, Hirsch HA, Struhl K. An epigenetic switch involving NF-kappaB, Lin28, Let-7 MicroRNA, and IL6 links inflammation to cell transformation. Cell 2009;139:693-706

27 Lau CC, Sun T, Ching AK, et al. Viral-human chimeric transcript predisposes risk to liver cancer development and progression. Cancer Cell 2014;25:335-49.

28 Zavattari P, Perra A, Menegon S, et al. Nrf2, but not beta-catenin, mutation represents an early event in rat hepatocarcinogenesis. Hepatology 2015;62:851-62
29 Laconi E, Oren R, Mukhopadhyay DK, et al. Long-term, near-total liver replacement by transplantation of isolated hepatocytes in rats treated with retrorsine. Am J Pathol 1998;153:319-29.

30 Guo D, Fu T, Nelson JA, et al. Liver repopulation after cell transplantation in mice treated with retrorsine and carbon tetrachloride. Transplantation 2002;73:1818-24.

31 Nagamachi A, Matsui $H_{\text {, Asou }} \mathrm{H}$, et al. Haploinsufficiency of SAMD9L, an endosome fusion facilitator, causes myeloid malignancies in mice mimicking human diseases with monosomy 7. Cancer cell 2013;24:305-17.

32 Runyan CE, Schnaper HW, Poncelet AC. The role of internalization in transforming growth factor beta1-induced Smad2 association with Smad anchor for receptor activation (SARA) and Smad2-dependent signaling in human mesangial cells. J Biol Chem 2005:280:8300-8

33 Hayes S. TGFbeta receptor internalization into EEA1-enriched early endosomes: role in signaling to Smad2. J Cell Biol 2002;158:1239-49.

34 Dennler $S$, André J, Alexaki I, et al. Induction of sonic hedgehog mediators by transforming growth factor-beta: Smad3-dependent activation of Gli2 and Gli1 expression in vitro and in vivo. Cancer Res 2007;67:6981-6.

35 Oike T, Komachi M, Ogiwara $H$, et al. C646, a selective small molecule inhibitor of histone acetyltransferase p300, radiosensitizes lung cancer cells by enhancing mitotic catastrophe. Radiother Oncol 2014;111:222-7.

36 Grivennikov SI, Greten FR, Karin M. Immunity, inflammation, and cancer. Cell 2010;140:883-99.

37 De Marzo AM, Platz EA, Sutcliffe $S$, et al. Inflammation in prostate carcinogenesis. Nat Rev Cancer 2007;7:256-69.

38 Farazi PA, DePinho RA. Hepatocellular carcinoma pathogenesis: from genes to environment. Nat Rev Cancer 2006:6:674-87.

39 Li L, Tang J, Zhang B, et al. Epigenetic modification of MiR-429 promotes liver tumour-initiating cell properties by targeting $\mathrm{Rb}$ binding protein 4 . Gut 2015;64:156-67

40 Snell LM, Brooks DG. New insights into type I interferon and the immunopathogenesis of persistent viral infections. Curr Opin Immunol 2015:34:91-8.

41 Teijaro JR, Ng C, Lee AM, et al. Persistent LCMV infection is controlled by blockade of type I interferon signaling. Science 2013;340:207-11.

42 Wilson EB, Yamada DH, Elsaesser $\mathrm{H}$, et al. Blockade of chronic type I interferon signaling to control persistent LCMV infection. Science 2013;340:202-7.

43 Hou J, Zhou Y, Zheng Y, et al. Hepatic RIG-I predicts survival and interferon- $\alpha$ therapeutic response in hepatocellular carcinoma. Cancer Cell 2014;25:49-63.

44 Fearon ER, Vogelstein B. A genetic model for colorectal tumorigenesis. Cell 1990;61:759-67

45 Land H, Parada LF, Weinberg RA. Cellular oncogenes and multistep carcinogenesis. Science 1983:222:771-8.

46 Hatziapostolou M, Polytarchou C, Aggelidou E, et al. An HNF4 $\alpha$-miRNA inflammatory feedback circuit regulates hepatocellular oncogenesis. Cell 2011;147:1233-47.

47 Clemens MJ. Targets and mechanisms for the regulation of translation in malignant transformation. Oncogene 2004;23:3180-8.

48 Mouw JK, Yui Y, Damiano L, et al. Tissue mechanics modulate microRNA-dependent PTEN expression to regulate malignant progression. Nat Med 2014;20:360-7.

49 Chang S, Wang RH, Akagi K, et al. Tumor suppressor BRCA1 epigenetically controls oncogenic microRNA-155. Nat Med 2011;17:1275-82. 